\title{
Optimal parametrization for the relativistic mean-field model of the nucleus
}

\author{
M. Rufa \\ Institut für Theoretische Physik, Universität Frankfurt, D-6000 Frankfurt am Main, Federal Republic of Germany \\ P.-G. Reinhard, ${ }^{*}$ J. A. Maruhn, ${ }^{\dagger}$ and W. Greiner ${ }^{\dagger}$ \\ Joint Institute for Heavy Ion Research, Oak Ridge, Tennessee 37831 \\ M. R. Strayer \\ Oak Ridge National Laboratory, Oak Ridge, Tennessee 37831
}

(Received 30 November 1987)

\begin{abstract}
We study a relativistic model of the nucleus consisting of nucleons coupled to mesonic degrees of freedom via an effective Lagrangian whose parameters are determined by a fit to selected nuclear ground-state data. We find that the model allows a very good description of nuclear ground-state properties. Because of the relativistic nature of the model, the spin properties are uniquely fixed. We discuss variations of the parametrization and of the data which suggest that the present fit has exhausted the limits of the mean-field approximation, and discuss extensions which go beyond the mean field.
\end{abstract}

\section{INTRODUCTION}

In this paper we investigate a relativistic mean-field theory of nuclear ground-state properties. Mean-field theories have been very successful in describing many aspects of the nuclear ground state and nuclear dynamics. The most prominent of these theories is probably the Hartree-Fock and time-dependent Hartree-Fock calculations using Skyrme forces; for reviews see Refs. 1-4. The Skyrme force is a zero-change, density, and momentumdependent effective interaction whose form can be derived from a density matrix expansion of the nuclear $G$ matrix. It allows one to describe, with a few parameters, bulk and single-particle properties of nuclei from ${ }^{16} \mathrm{O}$ to superheavy nuclei. The binding energies, radii, electromagnetic form factors at low momentum, giant resonance excitations, and fission and fusion barriers are all well described by the model. However, there are several features which are not so well treated by Skyrme forces. These concern spin properties. The spin-orbit force, which is necessary to get the proper magic nuclei, is introduced "by hand," requiring an extra parameter, and any further spin couplings are completely undetermined. This happens because the Skyrme force is a nonrelativistic model, and spin properties are genuine relativistic effects.

Relativistic mean-field theories have been investigated almost as long as their nonrelativistic counterparts ${ }^{5,6}$ and they have proven to be a flexible and powerful method $;^{7-13}$ for a review, see Ref. 14. There are noted advantages of this method: Firstly, nuclear saturation has already been achieved at the lowest level of the model without any extra density dependence, ${ }^{6}$ and secondly, the spin-orbit force is implicit in the model without any need for extra adjustment. A fair agreement of nuclear shapes from ${ }^{16} \mathrm{O}$ through ${ }^{208} \mathrm{~Pb}$ can be achieved by inclusion of the Coulomb force and an isovector meson $(\rho$ field $){ }^{8-10}$
With the inclusion of the nonlinear self-couplings of the scalar field, a good agreement can be achieved for the energies $^{7,12,13}$ and also the nuclear compressibility. The full model reaches the high level of quality of its successful nonrelativistic counterparts. ${ }^{13}$ Studies on nucleonnucleus scattering also show that spin and polarization properties are very naturally described by a relativistic optical potential at a mean-field level. ${ }^{15}$ A relativistic treatment has also been very successful in a scheme which goes beyond the mean field; namely, in a Brueckner-Hartree-Fock model which accounts for twonucleon correlations. Here it is possible to describe the binding energy and the saturation density of nuclear matter within the ladder approximation of the manybody diagrammatic analysis. ${ }^{16,17}$

In a relativistic mean-field theory, we describe nucleons by the Dirac equation, and the interaction between the nucleons is mediated by mesons. The Lagrangian of the model is an effective Lagrangian in the same sense as the Skyrme force is an effective force for nonrelativistic mean-field calculations. The difference is that there is a well-developed scheme to justify the form of the Skyrme force from nonrelativistic many-body theory. ${ }^{18}$ However, in the relativistic scheme, one is missing, up to now, clear guidance as to how to map the many-body effects and the quantum field effects into an effective Lagrangian of mesonic degrees of freedom and nucleons. ${ }^{14,19}$ The selection of the mesons is inspired by the one-boson-exchange models of the nucleon-nucleon interaction. ${ }^{20}$ This, quite naturally, leads to a Lagrangian which is renormalizable and which looks like a basic field theoretical Lagrangian; however, these parameters are adjusted to describe as many nuclear data as possible within a mean-field treatment.

It is our aim in this paper to study extensively the effects of the various meson parameters and look for the connection to one-boson-exchange models. We fit the model parameters to experimentally observable hulk 
properties of the nuclear ground state: binding energy, radius, and surface thickness. Other observables and nuclear matter properties are also considered; in the language of least-squares fits, we consider them as extrapolated quantities. The procedure closely follows comparable investigations with the Skyrme-force model, ${ }^{21}$ and thus allows a direct comparison between the nonrelativistic and the relativistic approaches. Early results of the investigation within the relativistic approach have been published in Ref. 22.

The paper is organized as follows. In Sec. II we present the Lagrangian, explain the approximations involved and derive the coupled field equations of the model. In Sec. III we describe the evaluation of the nuclear diffraction radius and surface thickness. In Sec. IV we explain the least-squares techniques and the selection of fit observables. In Sec. V we discuss other observables that are not directly used in the least-squares fits and nuclear matter properties. Finally, in Sec. VI we present the results of the practical investigations.

\section{THE FIELD EQUATIONS}

In the relativistic mean-field model of the nucleus, the nucleons are described by the Dirac equation, and the forces are generalized to be mesonic degrees of freedom. In practice, we start from the Lagrangian density

$$
\begin{aligned}
\mathcal{L}= & \bar{\psi}\left(i \gamma^{\mu} \partial_{\mu}-m_{B}\right) \psi+\frac{1}{2}\left(\partial^{\mu} \Phi \partial_{\mu}-m_{\sigma}^{2} \Phi^{2}\right)-\frac{1}{2}\left(\partial^{\tilde{v}} V^{\mu} \partial_{v} V_{\mu}-m_{\omega}^{2} V^{\mu} V_{\mu}\right)-\frac{1}{2}\left(\partial^{\tilde{v}} R^{\mu} \cdot \partial_{v} R_{\mu}-m_{\rho}^{2} R^{\mu} \cdot R_{\mu}\right) \\
& -\frac{1}{2} \partial^{\widetilde{v}} A^{\mu} \partial_{v} A_{\mu}-g_{\sigma} \Phi_{\bar{\psi} \psi}-g_{\omega} V^{\mu} \bar{\psi} \gamma_{\mu} \psi-\frac{1}{2} g_{\rho} R^{\mu} \cdot \bar{\psi} \tau \gamma_{\mu} \psi \\
& -e A^{\mu} \bar{\psi} \frac{1+\tau_{0}}{2} \gamma_{\mu} \psi-\frac{b_{2}}{3} \Phi^{3}-\frac{b_{3}}{4} \Phi^{4}-\frac{f_{\omega}}{4 m_{B}} \partial^{v} V^{\mu} \bar{\psi} i \gamma_{\mu} \gamma_{v} \psi-\frac{f_{\rho}}{8 m_{B}} \partial^{v} R^{\mu} \cdot \bar{\psi} \tau i \gamma_{\mu} \gamma_{v} \psi
\end{aligned}
$$

where the tilde denotes $\partial^{\tilde{v}} V^{\mu}=\partial^{v} V^{\mu}-\partial^{\mu} V^{v}$. The notation follows that of Refs. 23 and 24. This Lagrangian includes a scalar $(\sigma)$ meson field, $\Phi$, which provides the mediumrange attraction for the nucleons, a vector $(\omega)$ field, $V_{\mu}$, which describes the short-range repulsion, an isovector ( $\rho$ ) meson field, $R_{\mu}$, which is needed to adjust the isotropic properties of nuclei, and the electromagnetic field, $A_{\mu}$. For the coupling to the nucleons, we first take the simplest choice in each case; i.e., a scalar coupling $\left(g_{0}\right)$ to the scalar meson and a vector coupling $\left(g_{\omega}, g_{\rho}, e\right)$ to the vector mesons. This leads to a model which is linear in the fields. It proves to be insufficient for describing nuclear properties. Therefore we include a second- and third-order nonlinear self-coupling $\left(b_{2}, b_{3}\right)$ for the scalar field. It is interesting to note that, up to this stage, the theory is renormalizable (besides a few refinements which are necessary to make the $\rho$-coupling renormalizable but which drop out again in a static and spherically symmetric treatment). However, renormalizability is not a strong argument if one is dealing with effective theories. It happens just to be the simplest choice which gives reliable results. We shall note that further improvement is achieved by adding a tensor coupling $\left(f_{\omega}, f_{\rho}\right)$ for the vector mesons, leading to an extended model. The $\pi$ meson, which is the predominant and most long-ranged meson, is not included. It cannot contribute in a mean-field treatment of the ground state of even nuclei, because of conservation of parity.

The Lagrangian (1) is untractable on a full, quantal field level and treats the model within the mean-field approximation, wherein the meson fields are treated as simple, classical $c$-number fields. As a consequence, the nucleons move in classical fields as independent particles. Thus we can expand the field operator $\psi$ in terms of single-particle wave functions $\phi_{\alpha}$ :

$$
\psi=\sum_{\alpha} a_{\alpha} \phi_{\alpha}
$$

Using this, we can evaluate the nuclear currents, which are sources for the meson equations, in terms of the $\phi_{\alpha}$; for the scalar density we obtain

$$
\rho_{s}=\langle: \bar{\psi} \psi:\rangle=\frac{1}{2}\left(\sum_{\alpha=-\infty}^{F} \bar{\phi}_{\alpha} \phi_{\alpha}-\sum_{\alpha>F}^{+\infty} \bar{\phi}_{\alpha} \phi_{\alpha}\right),
$$

where the colons indicate the normal ordered product with respect to the ground state with Fermi level $F$. In Fig. 1 we show a typical nucleon spectrum in the presence of the meson fields. There is the negative energy continuum which provides the unbound antiparticle states. There are a few bound antiparticle states. Then there come the positive energy bound states; these correspond to the conventional nuclear shell model states and are the states in which we are most interested, because they mainly build up the nuclear mean field. Finally, there comes the particle continuum. As an example, we have drawn in Fig. 1 the Fermi surface $F$ which corresponds to an ${ }^{16} \mathrm{O}$ nucleus. One can imagine that the infinite summations involved in the density (3) are very hard to handle. In order to see what they contain, we rewrite the sums by introducing another Fermi surface $F_{0}$ which corresponds to a state with total baryon number 0; see Fig. 1. Thus

$$
\rho_{s}=\frac{1}{2}\left(\sum_{-\infty}^{F_{0}} \bar{\phi}_{\alpha} \phi_{\alpha}-\sum_{F_{0}}^{+\infty} \bar{\phi}_{\alpha} \phi_{\alpha}\right)+\sum_{\alpha=1}^{A} \bar{\phi}_{\alpha} \phi_{\alpha},
$$

where $A$ is the nucleon number contained between surfaces $F_{0}$ and $F$. The first term therein describes vacuum polarization effects, and the second term describes the 
bulk properties of the $A$ nucleons in the nucleus. As a second approximation, we neglect the vacuum polarization, which we call the no-sea approximation. Thus, we remain with a manageable expression for the nucleon currents, e.g.,

$$
\rho_{s}=\sum_{\alpha=1}^{A} \bar{\phi}_{\alpha} \phi_{\alpha},
$$

where the sum runs only over the few occupied nuclear shell-model states. In order to compute also nonmagnetic nuclei, we will extend the sum to a slightly larger valence space and allow for occupation numbers $w_{\alpha}$, where

$$
0 \leq w_{\alpha} \leq 1
$$

We now take the Lagrangian (1) with these approximations and perform the standard variational procedure. ${ }^{24}$ This leads to the coupled field equations. We write them down here in a form which is already specialized to stationary solutions; that is, where

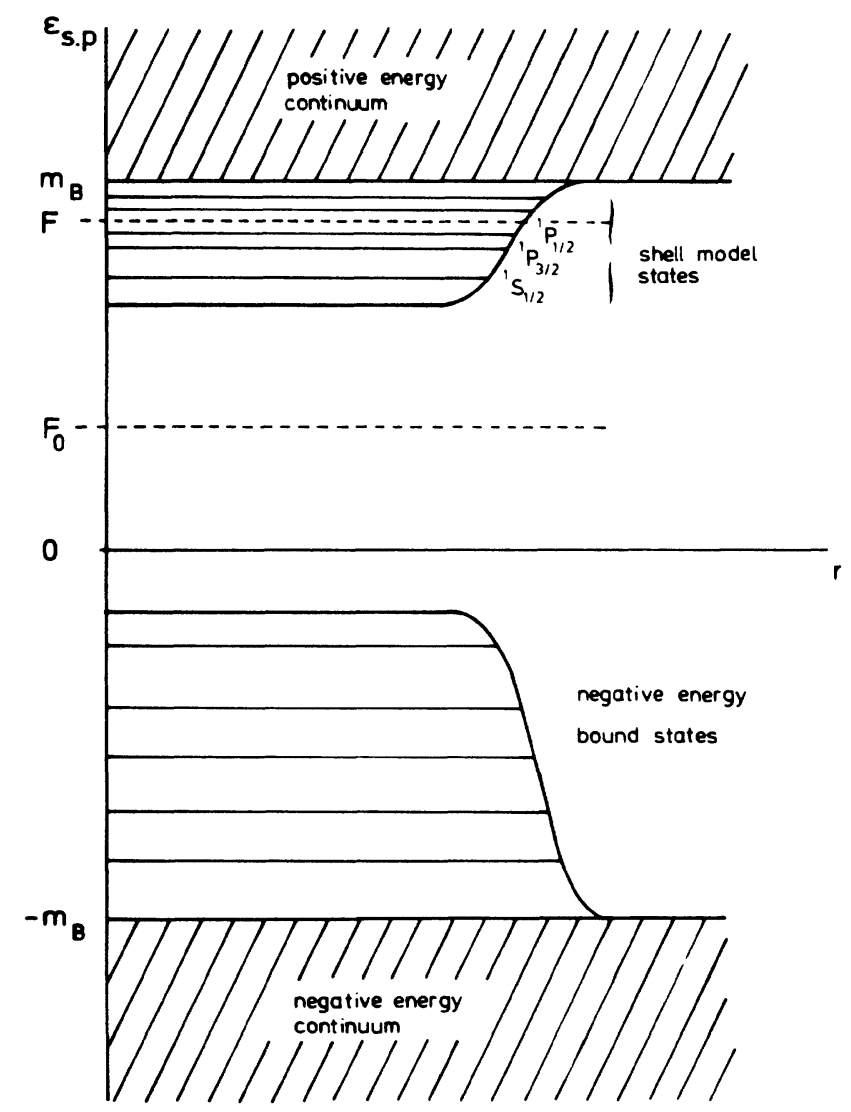

FIG. 1. Schematic spectrum of the Dirac equation for the nucleons. The positive, energy-bound states exhibit a one-toone correspondence to the states of a classical nuclear shell model. This is indicated by denoting these states with the usual spectroscopic quantum numbers. The potential for negativeenergy states can also support bound states as indicated. The Fermi surfaces $F$ for the ground state of ${ }^{16} \mathrm{O}$ and $F_{0}$ for the vacuum state (zero baryons) are indicated by dashed lines.

$$
\begin{aligned}
& i \partial_{t} \phi=\epsilon_{\alpha} \phi_{\alpha}, \\
& \partial_{t}^{2} \Phi=0, \\
& \partial_{t}^{2} V_{\mu}=0, \\
& V_{i}=0 \text { for } i=1,2,3,
\end{aligned}
$$

and similarly

$$
\begin{aligned}
A_{i} & =0, \\
R_{i} & =0 .
\end{aligned}
$$

We furthermore consider the case that all single-particle wave functions $\phi_{\alpha}$ have definite isospin, being either neutrons or protons. This yields the equations

$$
\begin{aligned}
& \epsilon_{\alpha} \gamma_{0} \phi_{\alpha}=\left(-i \gamma \cdot \nabla+m_{B}+g_{0} \Phi+g_{\omega} V_{0} \gamma_{0}+\frac{f_{\omega}}{2 m_{B}}\left(\nabla V_{0}\right) \cdot i \alpha\right. \\
& \quad+\frac{1}{2} g_{\rho} R_{00} \gamma_{0} \tau_{0}+\frac{f_{\rho}}{4 m_{B}} \nabla R_{00} \cdot i \alpha \tau_{0} \\
& \quad+\left.e A_{0} \gamma_{0} \frac{1+\tau_{0}}{2}\right|_{\alpha}, \\
& \left(-\Delta+m_{\sigma}^{2}\right) \Phi=-g_{\sigma} \rho_{s}-b_{2} \Phi^{2}-b_{3} \Phi^{3}, \\
& \left(-\Delta+m_{w}^{2}\right) V_{0}=g_{w} \rho_{0}-\frac{f_{w}}{2 m_{B}} \rho_{0}^{T}, \\
& \left(-\Delta+m_{\rho}^{2}\right) R_{00}=\frac{1}{2} g_{\rho} \rho_{00}-\frac{f_{\rho}}{4 m_{B}} \rho_{00}^{T}, \\
& -\Delta A_{0}=e \rho_{P, 0},
\end{aligned}
$$

with the densities given as

$$
\begin{aligned}
& \rho_{s}=\sum_{\alpha=1}^{\Omega} w_{\alpha} \bar{\phi}_{\alpha} \phi_{\alpha}, \\
& \rho_{0}=\sum_{\alpha=1}^{\Omega} w_{\alpha} \bar{\phi}_{\alpha} \gamma_{0} \phi_{\alpha}, \\
& \rho_{0}^{T}=\nabla \cdot \sum_{\alpha=1}^{\Omega} w_{\alpha} \bar{\phi}_{\alpha} i \alpha \phi_{\alpha}, \\
& \rho_{00}=\sum_{\alpha=1}^{\Omega} w_{\alpha} \bar{\phi}_{\alpha} \gamma_{0} \tau_{0} \phi_{\alpha}, \\
& \rho_{00}^{T}=\nabla \cdot \sum_{\alpha=1}^{\Omega} w_{\alpha} \bar{\phi}_{\alpha} i \alpha \tau_{0} \phi_{\alpha}, \\
& \rho_{P, 0}=\sum_{\alpha=1}^{\Omega} w_{\alpha} \bar{\phi}_{\alpha} \gamma_{0} \frac{1+\tau_{0}}{2} \phi_{\alpha} .
\end{aligned}
$$

The occupation weights $w_{\alpha}$ are still to be determined; we use a schematic pairing model with a constant gap $\Delta,{ }^{25}$ which gives

$$
w_{\alpha}=\frac{1}{2}\left(1+\frac{\epsilon_{\mathrm{Fermi}}-\epsilon_{\alpha}}{\left(\epsilon_{\mathrm{Fermi}}-\epsilon_{\alpha}\right)^{2}+\Delta^{2}}\right),
$$

where 


$$
\Delta=11.2 \mathrm{MeV} A^{-1 / 2}
$$

and $\epsilon_{\text {Fermi }}$ is to be adjusted such that $\Sigma w_{\alpha}$ reproduces the desired proton number or neutron number, respectively. The size of the valence space, $\Omega_{P}$ or $\Omega_{N}$, is chosen to include all occupied states up to the magic shell just being opened; thus, for magic nuclei there will be no pairing.
In practice, we are solving this set of coupled equations for spherical nuclei. This allows some further simplification since one only has to determine radial fields and wave functions. The details of the spherical reduction and of the numerical procedure are given in the Appendix. The energy for the system described by the Lagrangian (1) within the mean field and no-sea approximations is derived in the standard way. We obtain

$$
\begin{aligned}
E_{\mathrm{MF}}=\sum_{\alpha=1}^{\Omega} w_{\alpha} \epsilon_{\alpha}-\frac{1}{2} \int d^{3} r & {\left[g_{\sigma} \Phi \rho_{s}+\frac{1}{3} b_{2} \Phi^{3}+\frac{1}{2} b_{3} \Phi^{4}\right.} \\
& \left.+V_{0}\left(g_{\omega} \rho_{0}-\frac{f_{\omega}}{2 m_{B}} \rho^{T}, 0\right)+\frac{1}{2} R_{00}\left[g_{\rho} \rho_{00}-\frac{f_{\rho}}{2 m_{B}} \rho^{T}, 00\right]+e A_{0} \rho_{P, 0}\right],
\end{aligned}
$$

where the field terms of the type $\nabla^{\mu} \Phi \nabla_{\mu} \Phi$ have been eliminated by partial integration. The above expression is advantageous for the numerical integration, because it requires only quantities which are involved in solving the coupled field equations.

There is an additional contribution from the pairing energy

$$
E_{\mathrm{pair}}=-\frac{1}{2} \Delta \sum_{\alpha} w_{\alpha}\left(1-w_{\alpha}\right)
$$

which will be small compared to the above mean-field energy. Finally, we have to take into account a center-ofmass correction to the energy. As a simple approach to the center-of-mass projection, we subtract the expectation value of the center-of-mass energy

$$
E_{\mathrm{c} . \mathrm{m} .}=\frac{\left\langle P_{\mathrm{c} . \mathrm{m} .}^{2}\right\rangle}{2 m_{B} A},
$$

where

$$
\begin{aligned}
\left\langle P_{\mathrm{c} . \mathrm{m} .}^{2}\right\rangle= & \sum_{\alpha=1}^{\Omega} v_{\alpha}^{2}\left(\phi_{\alpha}\left|P^{2}\right| \phi_{\alpha}\right) \\
& -\sum_{\alpha=1}^{\Omega}\left(v_{\alpha}^{2} v_{\beta}^{2}+v_{\alpha} v_{\beta} u_{\alpha} u_{\beta}\right)\left|\left(\phi_{\alpha}|P| \phi_{\beta}\right)\right|^{2},
\end{aligned}
$$

$v_{\alpha}=\omega_{\alpha}, u_{\alpha}=1-\omega_{\alpha}$, and the $\left(\phi_{\alpha}|\cdots| \phi_{\beta}\right)$ are singleparticle matrix elements. This is a nonrelativistic centerof-mass correction taken over from a comparable nonrelativistic mean-field model. ${ }^{21}$ We assume that it is sufficient since the motion of the centel of-mass will be slow. Putting all the contributions $[(8)-(10)]$ together, we obtain the total binding energy

$$
E_{B}=E_{\mathrm{MF}}+E_{\mathrm{pair}}-E_{\mathrm{c} . \mathrm{m} .}-A m_{B},
$$

where $A$ times the nucleon mass has been subtracted in order to give the binding energy.

The whole procedure outlined above looks, at first glance, very fundamental; we start from nucleons and mesons, do a few approximations, and derive coupled field equations by standard variational techniques. How- ever, the mean-field and no-Fermi-sea approximations implied are very dramatic. They neglect important many-body effects as the exchange terms and short-range correlations, and they neglect equally important quantum field effects such as nucleonic and mesonic vacuum polarization, coupling renormalization and mass renormalization. If one is going to estimate these effects for a reasonable parametrization, each one would contribute "corrections" at the $100 \%$ level. On the other hand, there must be, as the zeroth order, an underlying mean field which serves, at least, as an expansion basis for the further correlations and for which, moreover, rich experimental evidence of shell structure exists. The situation is comparable to the nonrelativistic case, where we know that the nucleon-nucleon interaction requires a diligent Brueckner-Hartree-Fock treatment. Nonetheless, a nuclear shell model emerges at the end, with the Brueckner $T$ matrix serving as an effective force for the mean-field calculations. ${ }^{26}$ These Brueckner-Hartree-Fock calculations never have led to a quantitative agreement with experimental data of nuclear bulk properties. ${ }^{27}$ Therefore, one has cut off the lines to the microscopic history of the $T$ matrix and parametrize it in terms of an effective force. The form of the effective force is usually motivated by a density matrix expansion of the $T$ matrix. ${ }^{18}$ This has led to the Skyrme forces which have been extremely successful in describing nuclear properties. ${ }^{1-4}$ We adapt the point of view that the Lagrangian (1) is an effective Lagrangian for relativistic calculations within the mean field and no-sea approximations, in the same sense as the Skyrme force is an effective Lagrangian for classical mean-field calculations. Thus, the mesons are effective mesons, and their parameters are considered as being the free parameters of the model to be adjusted to reproduce nuclear bulk properties as well as possible. From the parameters in the Lagrangian (1), the baryon mass is fixed at the free nucleon mass because there is no mass renormalization in the mean-field approximation. We take for both nucleons the same average mass, $m_{B}=938.7 \mathrm{MeV}$. The electromagnetic coupling is fixed because there is no charge renormalization, and we wish to obtain the correct asymptotic Coulomb field. Thus, we take $e^{2}=0.00730$. The masses of the $\omega$ and $\rho$ mesons, $m_{\omega}$ and 
$m_{\rho}$, turn out to have little influence on the results, provided the $\sigma$ mass is readjusted properly. We keep these fixed at the empirical values,

$$
\begin{aligned}
& m_{\omega}=780 \mathrm{MeV}=3.95 \mathrm{fm}^{-1}, \\
& m_{\rho}=763 \mathrm{MeV}=3.87 \mathrm{fm}^{-1} .
\end{aligned}
$$

There remain the following free parameters in the various stages of the model

$$
\begin{aligned}
& \text { Linear: } m_{\sigma}, g_{\sigma}, g_{\omega}, g_{\rho}, \\
& \text { Nonlinear: } m_{\sigma}, g_{\sigma}, g_{\omega}, g_{\rho}, b_{2}, b_{3}, \\
& \text { Extended: } m_{\sigma}, g_{\sigma}, g_{\omega}, g_{\rho}, b_{2}, b_{3}, f_{\omega}, f_{\rho} .
\end{aligned}
$$

We shall describe in Sec. V how these parameters are determined. The linear model is distinguished by its simplicity. One can hardly imagine how to do it differently. There is, however, some prejudice in selecting the mesons: they are chosen according to their importance in the OBEP's. The addition of the nonlinear terms is a bit more ambiguous. We want to implement some density dependence. That could be done in many different ways, since a relativistic theory gives us a variety of Lorentz invariants. The above choice, again, is the most simple, and it reflects some influence from field theory, since it still provides a renormalizable Lagrangian. The choice of the tensor couplings for the extended model may be even more ambiguous. Some momentum dependence is needed, and the tensor couplings are suggested because they appear already in the OBEP's. ${ }^{20}$ However, we will find that the values for the couplings $f_{w}$ and $f_{\rho}$ are significantly different from the OBEP values, and that no significant improvement is gained from the tensor couplings. Here we come to a point where the refinement of the model can proceed in so many ways that we need good theoretical reasons for including further terms.

There is another problem with the phenomenological adjustment of an effective Lagrangian. At some stage one has to determine the parameters of the effective Lagrangian directly from experiment. Thus, one has circumvented the explicit computation of two-body correlations. Correspondingly, we concentrate on observables which are not strongly influenced by correlations. In Sec. III we will explain how to evaluate the form factor and how to parametrize the low-momentum part of it by the diffraction radius and the surface thickness.

\section{ELECTROMAGNETIC FORM FACTORS}

Elastic electron scattering provides us with rather model-independent information on the nuclear shape. By now, there have been established clear-cut procedures to determine, from the measured cross section, a nuclear charge distribution and, accordingly, the charge form factor. ${ }^{28}$ We want to use these data in our adjustment of the meson parameters. To that end, we evaluate the charge form factor from the form factors of the nucleon densities within the nucleus,

$$
F_{x}(q)=4 \pi \int_{0}^{\infty} d r r^{2} j_{0}(q r) \rho_{x}(r)
$$

where we take into account the proton distribution $\rho_{p}=\frac{1}{2}\left(\rho_{0}+\rho_{00}\right)$, the neutron distribution $\rho_{n}=\frac{1}{2}\left(\rho_{0}-\rho_{00}\right)$, and their tensor currents $\rho_{T, x}=\frac{1}{2}\left(\rho_{x}^{T}, 0 \pm \rho_{x}^{T}, 00\right)$, for $x=p, n$. The full-charge form factor is superposed from these form factors multiplied by the intrinsic form factors of the nucleon, namely the charge form factor $f_{1}(q)$ and the form factor of the anomalous magnetic moment $f_{2}(q)$, yielding

$$
F_{C}(q)=\sum_{x=P, N}\left[F_{x}(q) f_{1, x}(q)+F_{T, x}(q) f_{2, x}(q)\right] \exp \left[+q^{2} /\left(8\left\langle P_{\mathrm{c} . \mathrm{m} .}^{2}\right\rangle\right)\right],
$$

where the overall exponential factor is the correction for the spurious center-of-mass motion, and where $\left\langle P_{\mathrm{c} . \mathrm{m} .}^{2}\right\rangle$ is the same momentum width as used in the energy correction $E_{\text {c.m. }}$, see Eq. (10).

The intrinsic nucleon form factors are taken from Ref. 29. They are given there in a classical reduction as the socalled Sachs form factors $G_{E}$ and $G_{M}$. The $f_{i, x}$ are related to these $G$ by ${ }^{30}$

$$
\begin{aligned}
& f_{1, x}=\left(\frac{1}{2} G_{E, I=0} \pm \frac{1}{2} G_{E, I=1}+\left(1+\mu_{x}\right) \frac{q^{2}}{4 m_{B}^{2}} G_{M}\right) /\left(1+\frac{q^{2}}{4 m_{B}^{2}}\right), \\
& f_{2, x}=\left(\left(1+\mu_{x}\right) G_{M}-\frac{1}{2}\left(G_{E, I=0} \pm G_{E, I=1}\right)\right) /\left(1+\frac{q^{2}}{4 m_{B}^{2}}\right),
\end{aligned}
$$

and $G$ are given by a four-term dipole fit

$$
G_{s}=\sum_{i=1}^{4} \frac{a_{s, i}}{1+q^{2} / b_{x, i}},
$$

where $s$ denotes $E, I=0$ or $E, I=1$, or $M$. The values for the coefficients are summarized in Table $I$. We want to remark that there is some ambiguity in choosing the intrinsic form factors off shell. We have chosen here the most naive point of view, using for the vector coupling simply $F_{1} A^{\mu} \gamma_{\mu}$, and for the tensor coupling $F_{2} \partial^{\mu} A^{v} \sigma_{\mu \nu}$.

As discussed in the previous Sec. II, we have to judge how sensitive the form factor will be to correlation effects. This depends on the transferred momentum $q$. Certainly, the region of higher $q$ values (above, say, twice the Fermi momentum) will be affected by various correlations. The low $q$ values will, probably, be rather insensitive, except perhaps for collective zero-point vibrations; 
TABLE I. The parameters for the intrinsic nucleon form factors. The $a_{i}$ are dimensionless and the $b_{i}$ are given in units of $\mathrm{fm}^{-2}$.

\begin{tabular}{lllllrlrr}
\hline \hline & $a_{1}$ & $a_{2}$ & \multicolumn{1}{c}{$a_{3}$} & $a_{4}$ & $b_{1}$ & $b_{2}$ & $b_{3}$ & $b_{4}$ \\
\hline$E, I=0$ & 2.2907 & -0.6777 & -0.7923 & 0.1793 & 15.75 & 26.68 & 41.04 & 134.2 \\
$E, I=1$ & 0.3681 & 1.2263 & -0.6316 & 0.0372 & 5.00 & 15.02 & 44.08 & 154.2 \\
$M$ & 0.694 & 0.719 & -0.418 & 0.005 & 8.50 & 15.02 & 44.08 & 355.4 \\
\hline \hline
\end{tabular}

thus we concentrate on the charge form factor $F_{C}(q)$ at low $q$. A typical form factor is shown as the solid line in Fig. 2. The overall form is that of a diffraction pattern which can be related to the scattering from a box of radius $R$. This diffraction radius $R$ can be determined by the first zero of the form factor $q_{0}^{(1)}$ :

$$
R=4.493 / q_{0}^{(1)}, \quad F_{C}\left(q_{0}^{(1)}\right)=0 .
$$

With that $R$, the form factor is usually described over a wide range of $q$. If one compares, however, the form factor of a box of radius $R$, the dashed line in Fig. 2 with a realistic form factor, one sees that the box form factor falls off much slower than the realistic one. This effect is due mainly to the infinite surface thickness of nuclei. We model the surface thickness by a folding with a Gaussian $\exp \left(-\frac{1}{2} q^{2} \sigma^{2}\right)$ and read off the surface thickness $\sigma$ from the suppression of the form factor at the position of the first maximum $q_{m}^{(1)}$ :

$$
\begin{aligned}
& \sigma=\frac{2}{q_{m}^{(1)}} \log \left(\frac{F_{\text {box }}\left(q_{m}^{(1)}\right)}{F_{C}\left(q_{m}^{(1)}\right)}\right), \\
& F_{\text {box }}(q)=3 \frac{j_{1}\left(q_{m} R\right)}{q_{m} R} .
\end{aligned}
$$

We consider these two observables, the diffraction radius $R$ and the surface thickness $\sigma$, to be the prominent features of the nuclear charge density, ${ }^{31}$ which we use as the source of information on the nuclear density in the fit of the meson parameters.

As argued above, it is rather probable that the lowmomentum portion, and with it $R$ and $\sigma$, of the form factor is not affected by short-range correlations. However, one knows that there are collective, low-energy excitations and surface vibrations which can have a substantial effect, especially on the surface thickness. ${ }^{32-34}$ We will consider, optionally, part of these collective correlations. In principle, one has to include models from $0^{+}$up to $4^{+}$ and $5^{-32}$ For simplicity and for just a first estimate of the influence of the collective correlations, we restrict ourselves to the best understood quadrupole surface vibrations. We start from the well-known correction to the rms radius

$$
r^{2}=r_{0}^{2}+r_{0}^{2} \frac{5}{4 \pi} \beta_{2}^{2},
$$

where $r$ is the rms, $r_{0}$ is the rms radius of the mean-field solution, and $\beta_{2}$ is the average quadrupole fluctuation which can be deduced from the $B(E 2)$ values. $^{32}$ Using the approximate relation between $R, \sigma$, and $r,{ }^{31}$

$$
r=\left(R^{2}+3 \sigma^{2}\right)^{1 / 2},
$$

we obtain the correction for $\sigma$,

$$
\sigma=\left(\sigma_{0}^{2}+r_{0}^{2} \frac{5}{12 \pi} \beta_{2}^{2}\right)^{1 / 2}
$$

In practice, we use this correction in the following way. We take the experimental $\sigma$, the experimental values for $\beta_{2}$, and also measured rms radii (within $r \simeq r_{0}$ ) and derive a correlation-corrected

$$
\sigma_{0}=\left(\sigma^{2}-\frac{1}{3} r^{2} 5 / 4 \pi \beta_{2}^{2}\right)^{1 / 2},
$$

which is used as the desired mean-field result for the surface thickness. It remains a task for further, more elaborate, investigations to consider the effects of other surface modes within the given effective Lagrangian.

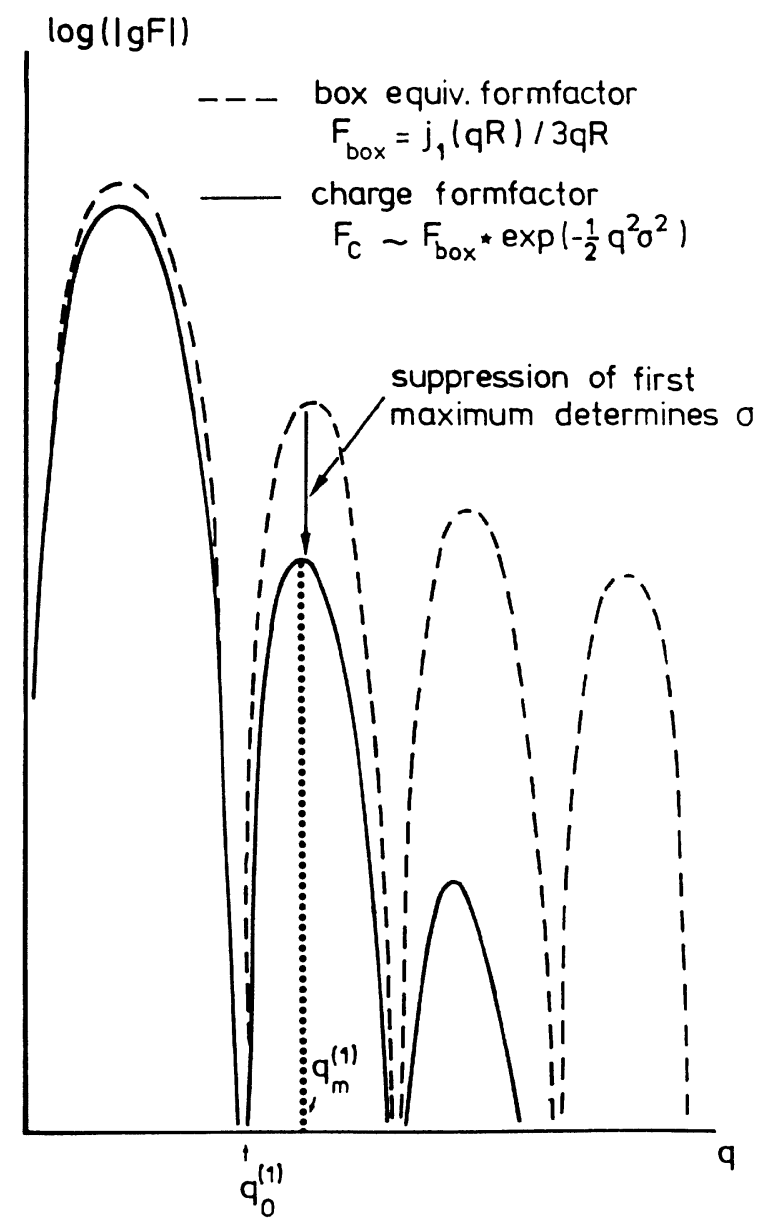

FIG. 2. Schematic plot of a nuclear charge form factor, its box equivalent form factor and the meaning of the form parameters $R$ and $\sigma$. 


\section{MODEL PARAMETERS}

As discussed in Sec. II, we consider the meson parameters as the adjustable parameters of an effective Lagrangian. We want to choose these parameters such that the model as stated reproduces the measured ground-state properties of nuclei. We do this, systematically, by minimizing the accumulated squared deviation,

$$
\chi^{2}=\sum_{n}\left(\frac{O_{n}^{\exp }-O_{n}^{\text {theory }}}{\Delta O_{n}}\right)^{2},
$$

with respect to the free parameters of the model. The sum in $\chi^{2}$ runs over all chosen observables $0_{n}$. In experimental data analysis, the $\Delta 0_{n}$ is the statistical error on the data. In our case, we cannot use just the experimental errors, because a mean-field theory is too crude to reproduce the nuclear bulk properties within the experimental resolution. The systematic error of the theory is too large. Therefore we insert, for $\Delta 0_{n}$, what we expect to be the ability of the theory to describe the observable $0_{n}$. The relation of the $\Delta 0_{n}$ 's, relative to each other, regulates the relative weights of the contributions to the $\chi^{2}$. We compute and compare the three observables, the binding energy $E_{B}$, the radius $R$ and the surface thickness $\sigma$ for the eight nuclei ${ }^{16} \mathrm{O},{ }^{40} \mathrm{Ca},{ }^{48} \mathrm{Ca},{ }^{58} \mathrm{Ni},{ }^{90} \mathrm{Zr}$, ${ }^{116} \mathrm{Sn},{ }^{124} \mathrm{Sn}$, and ${ }^{208} \mathrm{~Pb}$. These are all spherically symmetric nuclei. The set contains, with the two $\mathrm{Ca}$ nuclei and with the two Sn nuclei, some information on isotopic trends which may be related to the symmetry energy and other isovector properties. In Table II we give the experimental values for the $E_{B}, R$, and $\sigma$ in the eight nuclei. ${ }^{21}$ We also give the quadrupole fluctuation $\beta_{2}$ (Ref. 35) and the corrected surface thickness $\sigma_{0}$, according to Eq. (22). The selection of the observables, their error weights $\Delta 0_{n}$ and the nuclei, is the same as in similar fits of a nonrelativistic mean-field theory using Skyrme forces. ${ }^{21}$ This allows a close comparison of the nonrelativistic and the relativistic theory. Note, however, that we do not include extra information about the spin-orbit splitting here, as we did in the nonrelativistic case. A relativistic theory should predict this properly without further constraint.

For the minimization of the $\chi^{2}$, we use the CURFIT techniques, as described by Bevington. ${ }^{36}$ It is the advantage of the systematic $\chi^{2}$ techniques that one obtains not only the minimizing parameters, but also the uncertain- ties of it. There we distinguish between the uncorrelated error and the correlated error. Both errors describe the limits of an allowed variation of the parameters where $\chi^{2}$ remains within one unit of the value at the minimum. The uncorrelated error determines the allowed variation of one parameter, while all the others remain fixed. The correlated error determines the allowed variation of one parameter when all the others are readjusted to give minimal $\chi^{2}$. The $\chi^{2}$ techniques allow even more. One can estimate the uncertainties on extrapolated quantities, i.e., observables which had not been included in the fit. All these estimates use the curvature matrix

$$
a_{i j}=\frac{\partial^{2}\left(\chi^{2}\right)}{\partial p_{i} \partial p_{j}}
$$

where $p_{i}, p_{j}$ represent the free parameters of the model. The uncorrelated error is given by the diagonal curvature as $\delta p_{i}=\left[2\left(a_{i i}\right)^{-1}\right]^{1 / 2}$. The correlated error is given by the diagonal of the inverse curvature matrix as $\Delta p_{i}=2\left(a^{-1}\right)_{i i}$. The extrapolation error on any other observable $A$ is also derived from the inverse curvature matrix

$$
\Delta A=\left(2 \sum_{i, j} \frac{\partial A}{\partial p_{i}}\left(a^{-1}\right)_{i j} \frac{\partial A}{\partial p_{j}}\right)^{1 / 2}
$$

\section{OTHER OBSERVABLES}

We have taken care to include in the fit of the model parameters only such quantities which are directly accessible to experiment and which, moreover, one might hope are not obscured by correlation effects. There are other observables which do not meet these strict requirements but which are, nevertheless, useful to look at as, for example, the properties of nuclear matter. We start from the equation of state for symmetric nuclear matter, i.e., the energy per particle $E / A$ as a function of the baryon density $\rho_{0}$

$$
\begin{aligned}
E / A= & 4 \int_{0}^{k_{F}} d^{3} k k^{2}+\left(m_{B}+g_{0} \Phi\right)^{2}+\frac{1}{2} \frac{g_{\omega}^{2}}{m_{\omega}^{2}} \rho_{0} \\
& -\frac{1}{2} g_{\sigma} \Phi \rho_{s}-\frac{1}{6} b_{2} \Phi^{3}-\frac{1}{4} b_{3} \Phi^{4}
\end{aligned}
$$

TABLE II. The experimental values for the observables included in the fit. The $E_{B}, R$, and $\sigma$ are taken from Ref. 21, the $\beta_{2}$ from Ref. 35, and the $\sigma_{0}$ is computed from $\sigma$ and $\beta_{2}$ using Eq. (22) and $r=0.9 \mathrm{fm} \mathrm{A}^{1 / 3}$. In the last line we also give the adopted errors $\Delta O_{n}$ for the fit.

\begin{tabular}{rccccc}
\hline \hline & $E_{B}(\mathrm{MeV})$ & $R(\mathrm{fm})$ & $\sigma(\mathrm{fm})$ & $\beta_{2}$ & $\sigma_{0}(\mathrm{fm})$ \\
\hline${ }^{16} \mathrm{O}$ & -127.6 & 2.777 & 0.839 & 0.100 & 0.835 \\
${ }^{40} \mathrm{Ca}$ & -342.1 & 3.845 & 0.978 & 0.131 & 0.967 \\
${ }^{48} \mathrm{Ca}$ & -416.0 & 3.964 & 0.881 & 0.125 & 0.868 \\
${ }^{58} \mathrm{Ni}$ & -506.5 & 4.356 & 0.911 & 0.209 & 0.872 \\
${ }^{90} \mathrm{Zr}$ & -783.9 & 5.040 & 0.957 & 0.084 & 0.949 \\
${ }^{116} \mathrm{Sn}$ & -988.7 & 5.537 & 0.947 & 0.128 & 0.925 \\
${ }^{124} \mathrm{Sn}$ & -1050.0 & 5.640 & 0.908 & 0.107 & 0.891 \\
${ }^{208} \mathrm{~Pb}$ & -1636.4 & 6.806 & 0.900 & 0.084 & 0.885 \\
${ }_{0} 0_{n} / 0_{n}$ & $0.2 \%$ & $0.5 \%$ & $1.5 \%$ & & $1.5 \%$ \\
\hline \hline
\end{tabular}


where the scalar field is given by

$$
m_{\sigma}^{2} \Phi=-g_{\sigma} \rho_{s}-b_{2} \Phi^{2}-b_{3} \Phi^{3}
$$

and the scalar density

$$
\rho_{s}=4 \int_{0}^{k_{F}} d^{3} k \frac{m_{B}+g_{\sigma} \Phi}{k^{2}+\left(m_{B}+g_{\sigma} \Phi\right)^{2}},
$$

and the Fermi momentum $k_{F}$ is related to the baryon density

$$
k_{F}=\left(\frac{3 \pi^{2} \rho_{0}}{2}\right)^{1 / 3} .
$$

Equations (27a) and (27b) are combined to one transcendental equation determining $\Phi$ for a given $\rho_{0}, k_{F}$, respectively. With the $\Phi$ thus found, one can easily determine the $E / A$ according to Eq. (26).

Note that neither the Coulomb field, the $\rho$ meson, nor the tensor coupling play any role in the symmetric nuclear matter. Furthermore, the equation of state is insensitive to the meson masses. All dependences on the meson parameters can be reduced to the four-parameter combination

$$
\begin{aligned}
& C_{\sigma}^{2}=\frac{g_{\sigma}^{2} m_{B}^{2}}{m_{\sigma}^{2}}, \quad C_{\omega}^{2}=\frac{g_{\omega}^{2} m_{B}^{2}}{m_{\omega}^{2}}, \\
& B_{2}=\frac{b_{2}}{m_{\sigma} g_{\sigma}^{2}}, \quad B_{3}=\frac{b_{3}}{g_{\sigma}^{4}} .
\end{aligned}
$$

This is the combination of parameters which dominates the bulk binding energy also in finite nuclei. Therefore, we will consider these combinations in some of the studies following in Sec. VI.

From the equation of state, $E / A\left(\rho_{0}\right)$, we determine the ground state of symmetric nuclear matter by the equilibrium condition

$$
0=\frac{\partial(E / A)}{\partial \rho_{0}} .
$$

This yields the equilibrium density $\rho_{\mathrm{NM}}$ and, accordingly, the equilibrium energy $(E / A)_{\mathrm{NM}}$. A further, interesting quantity is the incompressibility $K_{\mathrm{NM}}$ which can be computed as usual

$$
K_{\mathrm{NM}}=9 \rho_{0}^{2} \frac{\partial^{2}}{\partial \rho_{0}^{2}}(E / A) .
$$

The resistance for similar vibrations of the proton against the neutron densities is measured by the symmetry energy $a_{4}$, which we evaluate directly by ${ }^{12}$

$$
a_{4}=\frac{k_{F}^{2}}{6\left[k_{F}^{2}+\left(m_{B}+g_{\sigma} \Phi\right)^{2}\right]^{1 / 2}}+\frac{1}{2} \frac{g_{\rho}^{2}}{m_{\rho}^{2}} \rho_{\mathrm{NM}} .
$$

Finally there is the effective mass
TABLE III. The experimental values of the pseudoobservables spin-orbit splitting $\sigma \epsilon_{\text {s.o. }}$, reciprocal level density $\Delta \epsilon$, and shell fluctuation $\sigma \epsilon$.

\begin{tabular}{lcccc}
\hline \hline$\epsilon_{\text {s.o. }}$ in & ${ }^{16} \mathrm{O}(\mathrm{MeV})$ & \multicolumn{1}{c}{$\Delta \epsilon$ in ${ }^{208} \mathrm{~Pb}(\mathrm{MeV})$} \\
Proton & Neutron & Proton & Neutron & $\delta \rho$ in ${ }^{208} \mathrm{~Pb}\left(\mathrm{fm}^{-3}\right)$ \\
\hline 5.98 & 6.07 & 1.086 & 1.057 & 0.0023 \\
\hline \hline
\end{tabular}

$$
m^{*}=m_{B}+g_{\sigma} \Phi,
$$

which is a critical quantity, as we will see in Sec. VI. There is some confusion about how to define an effective mass. Already in the nonrelativistic theory, there are at least three definitions from different physical viewpoints. The effective mass, as defined above, is the mass determining the motion of a very slow nucleon $(k \rightarrow 0)$ in the nuclear medium. It represents the value of the mass operator in the single-particle Green's function at $k \rightarrow 0$. This value is about $2-4 \%$ smaller than the $\left(\partial^{2} \epsilon / \partial k^{2}\right)^{-1}$ at the Fermi surface which is often considered as the effective mass. The $m^{*}$, according to Eq. (32), also represents the gap between nucleon and antinucleon solutions in the presence of nuclear matter. It is thus a rough estimate of this gap in finite nuclei.

We will also look at some properties in finite nuclei which we did not include in the least-squares fits. These are the spin-orbit splitting, the level density near the Fermi surface, and the shell fluctuations. The first two quantities have to do with the single-particle spectra, and the shell fluctuations are seen in the charge densities. For studies of trends and parameter dependences, we need to represent each of these features by one single number.

The strength of the spin-orbit force is read off from the splitting of the $1 p_{1 / 2}$ and the $1 p_{3 / 2}$ level in ${ }^{16} \mathrm{O}$

$$
\delta \epsilon_{\text {s.o. }}=\epsilon_{1 p_{1 / 2}}-\epsilon_{1 p_{3 / 2}},
$$

for protons or for neutrons, respectively. The experimental value for $\delta \epsilon_{\text {s.o. }}$ are given in Table III. They are deduced from the single-hole states with respect to ${ }^{16} \mathrm{O}$. It is known that these experimental quantities contain rearrangement corrections and effects from core polarization. In the case of ${ }^{16} \mathrm{O}$, the rearrangement effects are larger and the polarization effects are smaller. We expect that these effects, together, give an uncertainty of about 0.3 $\mathrm{MeV}$.

The level density near the Fermi surface is obtained from the last-filled proton or neutron shell in ${ }^{208} \mathrm{~Pb}$. We take the spreading width of the single-particle energies as a measure of the reciprocal level density,

$$
\Delta \epsilon=\left(\sum_{\alpha} \epsilon_{\alpha}^{2}-\sum_{\alpha} \epsilon_{\alpha}^{2}\right)^{1 / 2},
$$

where the sum runs over the last-filled shell in each case, i.e.,

$$
\alpha=\left\{\begin{array}{l}
3 s_{1 / 2}, 2 d_{3 / 2}, 2 d_{5 / 2}, 1 g_{7 / 2}, 1 h_{11 / 2} \text { for protons in }{ }^{208} \mathrm{~Pb} \\
3 p_{1 / 2}, 3 p_{3 / 2}, 2 f_{5 / 2}, 2 f_{7 / 2}, 1 h_{9 / 2}, 1 i_{13 / 2} \text { for neutrons in }{ }^{208} \mathrm{~Pb}
\end{array}\right.
$$




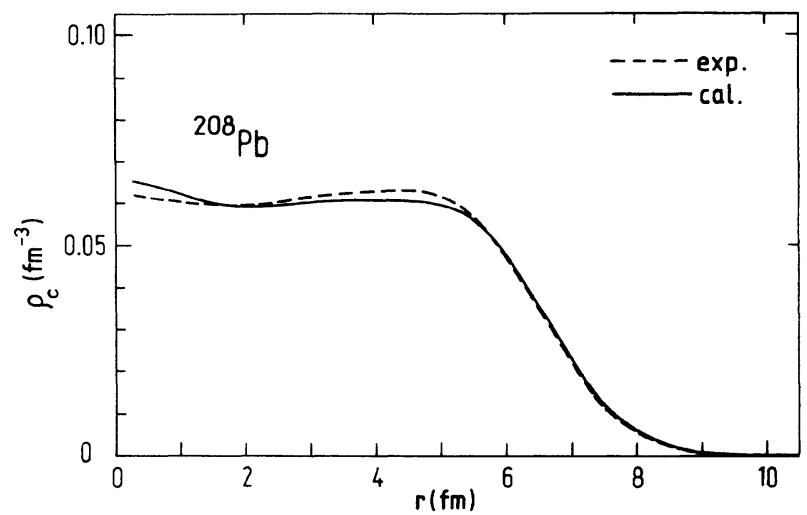

FIG. 3. The experimental charge density $\rho_{C}$ for ${ }^{208} \mathrm{~Pb}$ (dashed line) and the result from the relativistic mean-field model (full line, fit "nonlinear"). The pattern can be viewed as being composed of: first, a smooth Woods-Saxon background corresponding to radius $R$ and surface $\sigma$; second, a Coulomb repulsion of some density towards the surface; and third, an overall oscillation with wavelength $3.6 \mathrm{fm}$.

The experimental values are given in Table III. They are deduced from the single-hole states with respect to ${ }^{208} \mathrm{~Pb}$, as given in Ref. 37. Here the rearrangement effects may be very small, but the effects from core polarization are large due to the many low-lying collective states in ${ }^{208} \mathrm{~Pb}$. In the nonrelativistic calculations, the mean-field theory always produces single-particle spectra whose level density is, by a factor of 2, too small compared with the experimental value deduced from the single-hole spectra. ${ }^{21}$

The calculated charge density in nuclei deviates from a smooth Woods-Saxon distribution by a series of spatial oscillations with a typical wavelength of $3.6 \mathrm{fm}$. Depending on the shell filling, there is also a pronounced peak or dip at the nuclear center. ${ }^{38}$ As an example, we show in Fig. 3 the charge density of ${ }^{208} \mathrm{~Pb}$. These oscillations can be understood as a shell effect with a spatial wavelength of twice the Fermi momentum, the dominating momentum in the valence shell. The shell fluctuations are best obtained from the charge density in ${ }^{208} \mathrm{~Pb}$ as

$$
\delta \rho=\rho_{C}(0.0)-\rho_{C}(1.8 \mathrm{fm}) ;
$$

for an illustration see Fig. 3. The experimental value is given in Table III. The shell fluctuation $\delta \rho$ is related to the form factor at a momentum of $q=2 k_{F} \approx 2.7 \mathrm{fm}^{-1}$. Here the form factor will certainly be influenced by short-range correlations, mesonic exchange currents, giant resonances, and other correlations, and we know that the nonrelativistic mean-field theories overestimate $\delta \rho$ by more than a factor of $2 .^{21,38}$ Again, it is interesting to see how the relativistic mean-field theory behaves.

\section{RESULTS AND DISCUSSION}

\section{A. The linear model}

In a first step, we have optimized the four free parameters of the linear model, $g_{\sigma}, g_{\omega}, g_{\rho}$, and $m_{\sigma}$, to the standard set of data. We achieve an average precision of $2 \%$ for the energy, of $1.2 \%$ for the diffraction radius and of $23 \%$ for the surface thickness, adding up to a $\chi^{2}=2730$. This is a very bad reproduction of the data; in particular, the surface thicknesses are far too small. There are also strong misadjustments for some of the quantities not appearing in the $\chi^{2}$. In particular, the compressibility, $K=584 \mathrm{MeV}$, is far above commonly accepted values; also, the spin-orbit splitting in ${ }^{16} \mathrm{O}, \epsilon_{\text {s.o. }}=10 \mathrm{MeV}$, is far too large. The detailed figures can be found in the row labeled "Lin" in Table IV (parameters) and Table V (observables). Altogether, the linear model is inappropriate for a quantitative description of nuclear properties; one has to proceed to the nonlinear model.

\section{B. The nonlinear model}

In a second step, we optimize the six free parameters of the nonlinear model $g_{\sigma}, g_{\omega}, g_{\rho}, m_{\sigma}, b_{2}$, and $b_{3}$ to the standard set of data. This gives a much better fit with a precision of $0.32 \%$ for the energy, $0.70 \%$ for the diffraction radius, and $3.7 \%$ for the surface thickness, adding up to a $\chi^{2}=85$. This is comparable, in quality, to a similar fit within the Skyrme-Hartree-Fock description, ${ }^{21}$ showing that the relativistic mean-field theory is just as powerful as its nonrelativistic counterpart. We

TABLE IV. The dimensionless parameters according to Eqs. (28) for some of the sets in this paper. "NL- $Z$ " comes from the full fit to energy, radius, and surface. " $E$ and $R$ " comes from a restricted fit to energy and radius only. " $E$ " comes from a restricted fit to energy only. "CS" comes from a fit to energy, radius, and surface where the surface thickness has been corrected for contributions of the surface vibrations. "Lin" comes from a full fit with a restricted parametrization where the nonlinear self-couplings of the scalar meson have been switched off. "NL1" is the full fit from a previous publication (see Ref. 13); it is slightly different from "NL- $Z$ " here because a simplified zero-point energy has been used previously. "VT" is a full fit including a tensor coupling for the scalar meson (see Sec. VII). In all fits, the vector meson mass has been $780.00 \mathrm{MeV}$, except for the set "NLl" where it was $795.36 \mathrm{MeV}$. The isovector-vector meson mass has been $763.00 \mathrm{MeV}$ throughout.

\begin{tabular}{lcccccc}
\hline & $c_{s}^{2}$ & $c_{v}^{2}$ & $c_{R}^{2}$ & $B_{2}$ & $B_{3}$ & $m_{s}(\mathrm{MeV})$ \\
\hline NL-Z & 373.250 & 241.439 & 142.44 & $-0.279222 \times 10^{-2}$ & $-0.393463 \times 10^{-2}$ & 488.67 \\
$E$ and $R$ & 371.020 & 245.332 & 130.84 & $-0.237150 \times 10^{-2}$ & $-0.326815 \times 10^{-2}$ & 500.55 \\
$E$ & 370.575 & 245.570 & 123.53 & $-0.234809 \times 10^{-2}$ & $-0.325573 \times 10^{-2}$ & 502.71 \\
CS & 373.308 & 243.236 & 138.58 & $-0.265466 \times 10^{-2}$ & $-0.371885 \times 10^{-2}$ & 492.45 \\
Lin & 363.383 & 276.960 & 179.52 & & & 551.31 \\
NL1 & 373.176 & 245.458 & 149.67 & $-0.24578 \times 10^{-2}$ & $-0.34334 \times 10^{-2}$ & 492.25 \\
VT & 361.601 & 232.104 & 128.90 & $-0.294804 \times 10^{-2}$ & $-0.414921 \times \times^{-2}$ & 483.42 \\
\hline \hline
\end{tabular}


TABLE V. Nuclear matter properties and other observables for the parametrizations given in Table IV. The nuclear matter properties are the following: $E / A$ is the binding energy per particle, given in $\mathrm{MeV} ; \rho$ is the density, given in $\mathrm{fm}^{-3} ; \mathrm{K}_{\mathrm{the}}$ incompressibility, given in MeV; $m^{*} / m$ is the effective mass; $a_{4}$ is the symmetry energy, given in MeV. The other observables are the following: $\delta \epsilon$ is the spin-orbit splitting of the $1 p$ level in ${ }^{16} \mathrm{O}$ [see Eq. (32)] for both protons and neutrons, given in $\mathrm{MeV} ; \delta \rho$ the width of level distribution in the valence shells of ${ }^{208} \mathrm{~Pb}$ [see Eqs. (34)], for $x=$ proton or $x=$ neutron, given in $\mathrm{MeV} ; \delta \rho$ is 100 times the amplitude of the shell fluctuations in ${ }^{208} \mathrm{~Pb}$ [see Eq. (35)], given in $\mathrm{fm}^{-3}$.

\begin{tabular}{|c|c|c|c|c|c|c|c|c|c|c|}
\hline & $E / A$ & $\rho$ & $K$ & $m^{*} / m$ & $a_{4}$ & $\delta \epsilon_{P}$ & $\delta \epsilon_{N}$ & $\Delta \epsilon_{P}$ & $\Delta \epsilon_{N}$ & $\delta \rho$ \\
\hline NL-Z & $\begin{array}{r}-16.185 \\
(0.047)\end{array}$ & $\begin{array}{c}0.1509 \\
(0.0007)\end{array}$ & $\begin{array}{r}173.5 \\
(7.4)\end{array}$ & $\begin{array}{c}0.5830 \\
(0.0028)\end{array}$ & $\begin{array}{c}41.8 \\
(1.1)\end{array}$ & 5.79 & 5.87 & 2.06 & 1.61 & -0.63 \\
\hline$E$ and $E$ & -16.162 & 0.1489 & 221.1 & 0.5826 & 39.4 & 6.47 & 6.56 & 2.16 & 1.67 & -0.67 \\
\hline$E$ & -16.138 & 0.1501 & 222.0 & 0.5788 & 38.6 & 6.70 & 6.22 & 2.17 & 1.68 & -0.68 \\
\hline $\mathrm{CS}$ & -16.171 & 0.1501 & 187.9 & 0.5826 & 40.9 & 6.02 & 6.10 & 2.07 & 1.61 & -0.63 \\
\hline Lin & -17.071 & 0.1492 & 584.4 & 0.5318 & 48.8 & 10.01 & 10.11 & 3.19 & 2.38 & -0.73 \\
\hline NL1 & -16.43 & 0.1542 & 212.0 & 0.5709 & 43.6 & 6.06 & 6.12 & 2.07 & 1.56 & -0.61 \\
\hline VT & -16.088 & 0.1531 & 173.3 & 0.5918 & 39.8 & 5.32 & 5.39 & 2.06 & 1.66 & -0.62 \\
\hline
\end{tabular}

will call this optimal nonlinear parametrization "set NL$Z$," and we will discuss some results for this set in more detail. The $Z$ indicates the consistent zero-point energy (10), as distinguished from the previous fit, ${ }^{22}$ called here in NL1. The charge density from set NL- $Z$ was already plotted and compared with experiment ${ }^{31}$ in Fig. 3. It obviously provides a good reproduction of the overall features of the density, except for the shell fluctuations $\delta \rho$ which are, by a factor of 2.5 , too large. Set NL-Z and all further fits in this section share this disease with the nonrelativistic mean-field theories. ${ }^{21}$ One very probably has to go beyond the mean field in order to properly describe this quantity. In Fig. 4 we show the single-particle levels in ${ }^{208} \mathrm{~Pb}$ for the set $\mathrm{NL}-Z$ in comparison with experiment $^{37}$ and results from Skryme-Hartree-Fock. ${ }^{21}$ The shell closure and the rough level ordering are reproduced very well. However, the level density in the last occupied and in the first unoccupied shells is too small. This is very well expressed by $\Delta \epsilon$, the level width of the last occupied shell. Set NL-Z gives $\Delta \epsilon=2 \mathrm{MeV}$, and the experiment gives $\Delta \epsilon=1 \mathrm{MeV}$. Again, the relativistic mean-field theory shares this problem with the nonrelativistic case, and again, we know that correlation effects are responsible for the deviation from experiment. ${ }^{39}$

In Fig. 5, we show the single-particle levels in ${ }^{16} \mathrm{O}$ for set $\mathrm{NL}-Z$ in comparison with experiment ${ }^{37}$ and results from Skyrme-Hartree-Fock. ${ }^{21}$ We are interested here, in particular, in the spin-orbit splitting of the $1 p$ level. Obviously, it is reproduced very well. Note that this is achieved without the need to adjust an extra parameter, as was the case for the Skyrme forces.

The fit included the data of eight nuclei, but we see that the fit gives a good overall description of other nuclei. This is illustrated in Fig. 6. There obviously remain interesting trends within the isotopic chains which are not resolved by the model. The same behavior occurs in the nonrelativistic models, ${ }^{21}$ and again we have to consider effects beyond mean field. The trends very probably are due to low-energy collective modes. ${ }^{32-34}$ These are known to have a strong influence on the form parameters $R$ and $\sigma$.

It is clear that we cannot discuss all variations of the data and of the parametrization that follow in such detail as in Fig. 6. The discussion of spectral properties is reduced to a discussion of $\delta \epsilon_{\text {s.o. }}$ and $\Delta \epsilon$, as introduced in Sec. V. The discussion of the charge density is reduced to the form parameters $R, \sigma$, and the $\delta \rho$. The distribution of the errors on $E, R$, and $\sigma$ displayed in Fig. 6 is discussed in terms of an average error

$$
\overline{\text { Obs. }}=\sum \text { (Obs. }^{\text {exp }} \text {-Obs. }{ }^{\text {theory }} \text { ) }
$$

and of a rms error

$$
\left.\sqrt{\overline{\text { Obs. }^{2}}}=\left[\sum \text { (Obs. }^{\text {exp }}-\text { Obs. }^{\text {theory }}\right)^{2}\right)^{1 / 2} \text {, }
$$

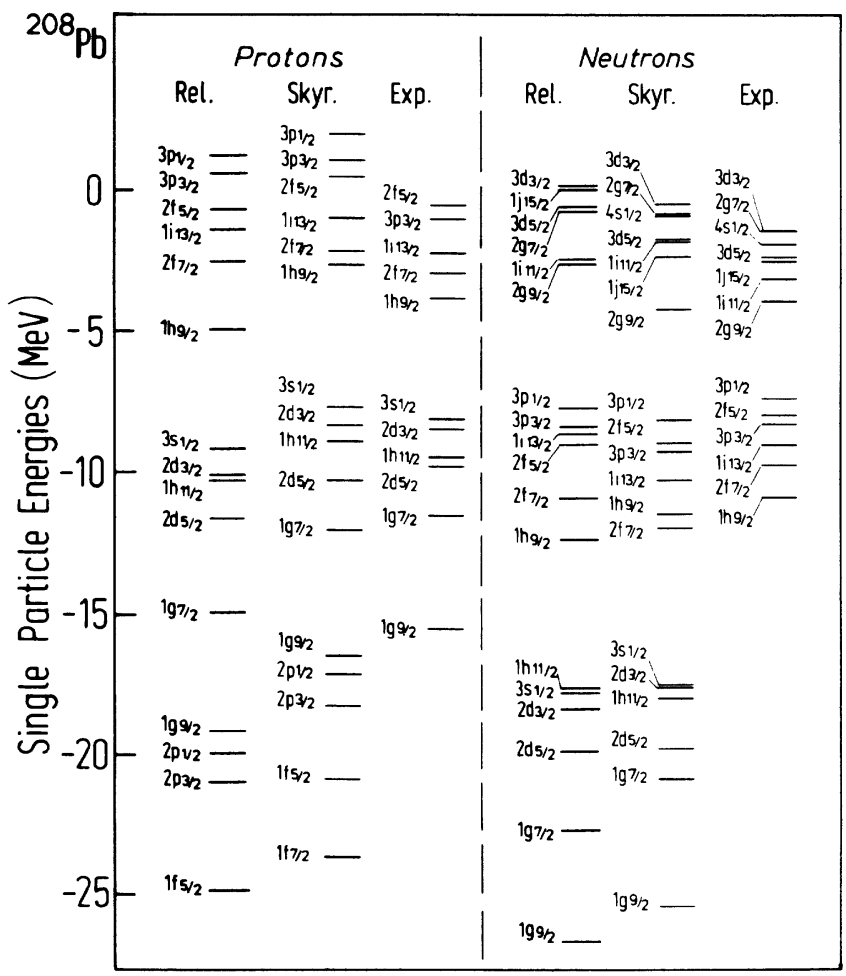

FIG. 4. The proton and neutron single-particle spectra for ${ }^{208} \mathrm{~Pb}$. We compare the results from the relativistic model, set NL, with Skyrme-Hartree-Fock (fit $Z_{0}$ from Ref. 21) and with experimental spectra. 


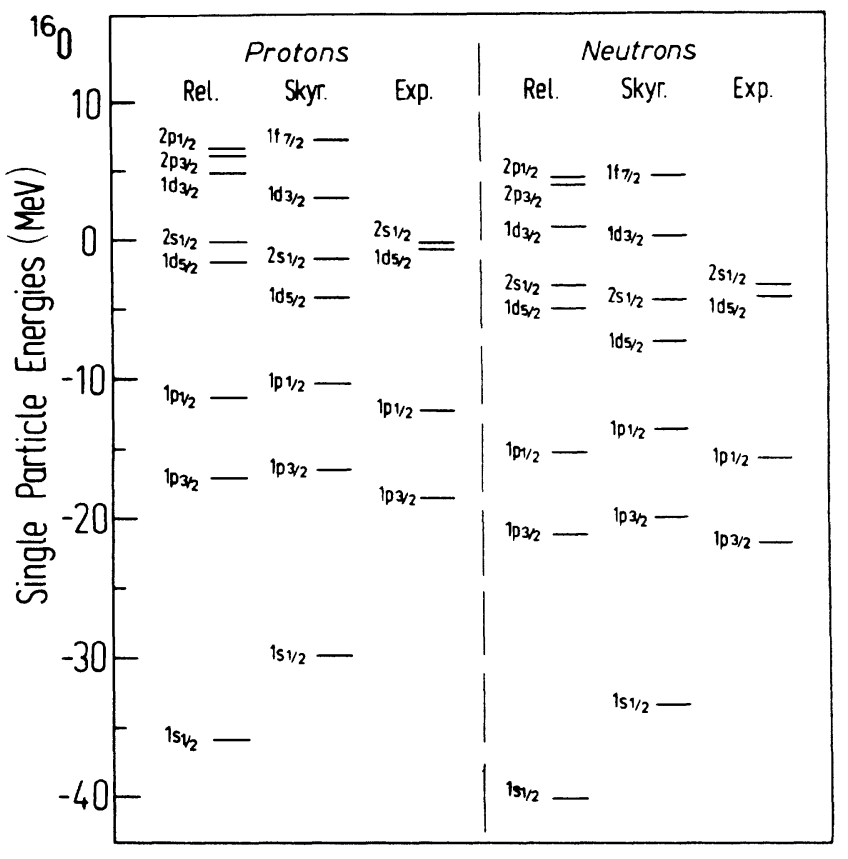

FIG. 5. The proton and neutron single-particle spectra for ${ }^{16} \mathrm{O}$. We compare the results from the relativistic model, set NL-Z, with Skyrme-Hartree-Fock (fit $Z_{0}$ from Ref. 21) and with experimental spectra.

where Obs. can be $E, R$, or $\sigma$, and the sum runs over the eight nuclei included in the fit. The average error displays unresolved trends, and the rms error shows the quality of the overall fit. Note that it is the rms error squared which enters, with the chosen weight, in the $\chi^{2}$.

\section{Variations of the data}

In order to check the sensitivity to the various observables included in the fit, we consider alternative fits of the nonlinear model with less data included, namely, first, a fit including only the energy $E$ and the diffraction radius $R$, and second, a fit including only the energy $E$. In Fig. 7 , we show the average and rms errors on $E, R$, and $\sigma$ for the various data selections. If we compare set $E$ and $R$ with set NL-Z, we see that $E$ and $R$ are indeed improved. The reproduction of $\sigma$, of course, gets worse. It is important to note that this is due mainly to a shift of the average error in $\sigma$; set $E$ and $R$ underestimates the surface thickness for all nuclei by about $5 \%$. This trend persists if we proceed to set $E$. Here it is rather surprising that the radius $R$ is still well reproduced, although it was not included in the fit.

Thus, we have seen that the relativistic mean-field model would like to produce less diffuse surfaces. We know that there are correlations from collective surface modes which smooth the surface. ${ }^{32-34}$ As discussed in Sec. IV, we can implement a phenomenological correction to $\sigma$ coming from the quadrupole surface vibrations. The corrected values, $\sigma_{0}$, are shown in Table II. We have included a fit comprised of all data, but using the surface-corrected $\sigma_{0}$. The resulting errors on the observ-
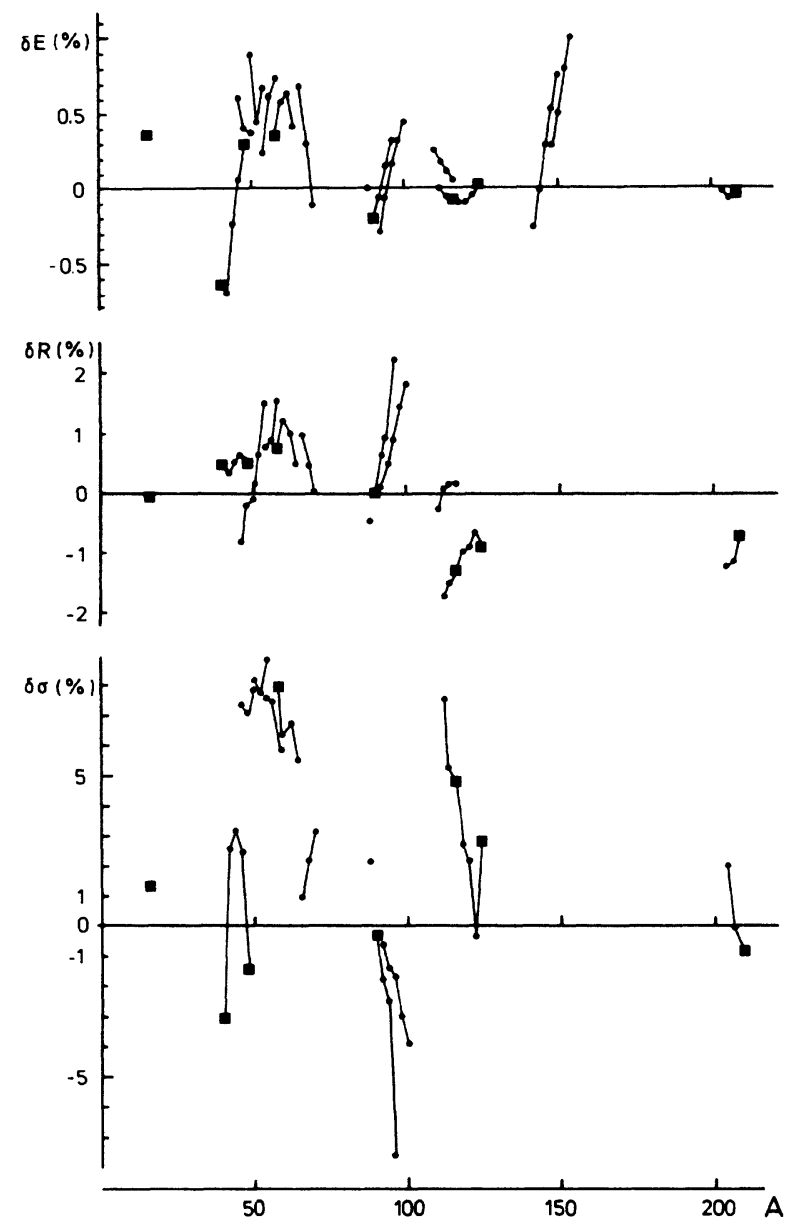

FIG. 6. The relative errors for energy $E$, diffraction radius $R$, and surface thickness $\sigma$ for a variety of nuclei. The results are drawn vs mass number $A$. Isotopic chains are connected by a line. The 8 nuclei included in the fit are distinguished by an extra circle around the dot.

ables are given in Fig. 7. We see that set $\mathrm{CS}$ gives some improvement compared to set $\mathrm{NL}-Z$, in particular with respect to surface properties. This shows that the correction goes in the right direction. However, more surface modes need to be included to make up a consistent picture. $^{34}$ It is interesting to note that the Skyrme forces are more flexible to fit the uncorrected $\sigma$, see row Sk. in Fig. 7.

In Table IV, we present observables calculated for the various parameter sets discussed above. The nuclear matter properties are also given with their extrapolation errors. The linear parametrization differs dramatically in all its predictions from any other set and from commonly believed reasonable values. It is clearly ruled out. There are only small differences between the predictions of the other parametrizations. Comparing Skyrme results ${ }^{21}$ with liquid drop values, ${ }^{40}$ we see that the relativistic models tend to give somewhat larger binding energies, somewhat lower densities, and rather large symmetry energies.

In Table $\mathrm{V}$, we give the corresponding parameter sets, since all parametrizations discussed up to now may be useful for other investigations. 


\section{Fits close to $\mathrm{OBE}$ potentials}

As discussed in previous sections, we do not yet know how to draw the connections from a microscopic picture of the nucleon-nucleon interaction to the effective meanfield Lagrangian. Nevertheless, it is interesting to ask how close the mean-field parametrization can be to OBE potentials. We take the point of view that the $\sigma$ meson is the most unphysical meson, and that the $\omega$ and $\rho$ mesons should come quite close to those in the OBE potentials. Thus, we take some current OBEP's, keep the $\rho$ and the $\omega$ meson parameters fixed, and fit only the four $\sigma$ meson parameters $g_{\sigma}, b_{2}, b_{3}$, and $m_{\sigma}$. In particular, we discuss in the following set HEA from Ref. 41, called OBE1, set EHM from Ref. 41, called OBE2, and the set from Ref. 20, called OBE3.

In Fig. 8, we present the average and rms errors for the OBE fits in comparison with the optimal set NL-Z. Obviously, there are differences in the quality of the fits be-
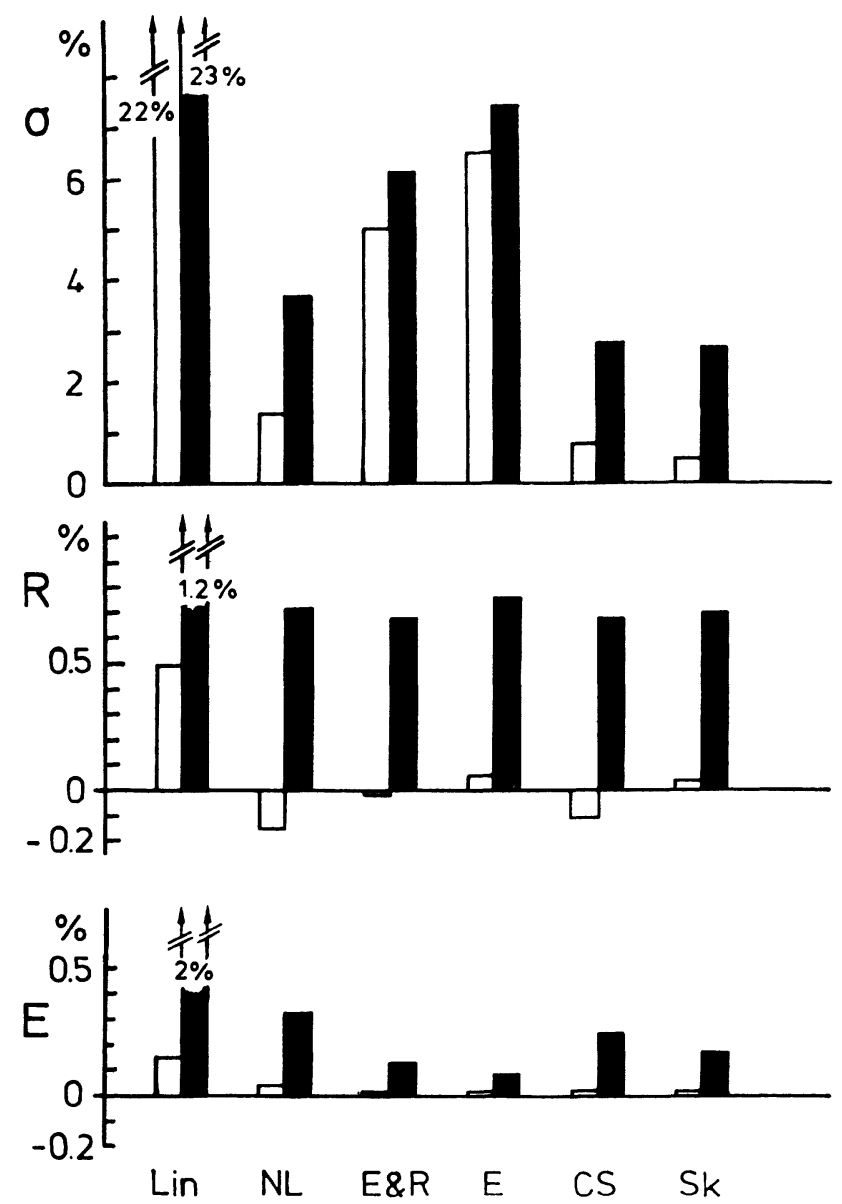

FIG. 7. The average errors (open cases) and the rms errors (shaded cases) for the observables $E, R$, and $\sigma$ and for various sets: Lin denotes the linear model fitted to full data; NL the NL- $Z$ nonlinear model fitted to full data; $E$ and $R$ the nonlinear model fitted to $E$ and $R$ only; $E$ the nonlinear model fitted to $E$ only; CS the nonlinear model fitted to full data with $\sigma$ corrected for surface vibrations; Sk the result of Skyrme force fit for comparison. tween the three OBEP's. However, there is at least one surprisingly good fit, OBE1, showing that indeed there is still some relation of the effective Lagrangian to the more microscopic OBEP's. It is interesting to note that the quality of the fits is related to the strength of the $\rho$ coupling. The relativistic mean-field theory obviously likes to have large $\rho$ couplings. We will discuss the $\rho$ coupling in Sec. VI E.

\section{E. The effect of the $\rho$ meson}

The above studies of OBE-related sets hint that a sizable $\rho$ coupling is needed for a good fit to the binding energies. We now study the effect of the $\rho$ meson separately by examining fits within the nonlinear model for a fixed $\rho$
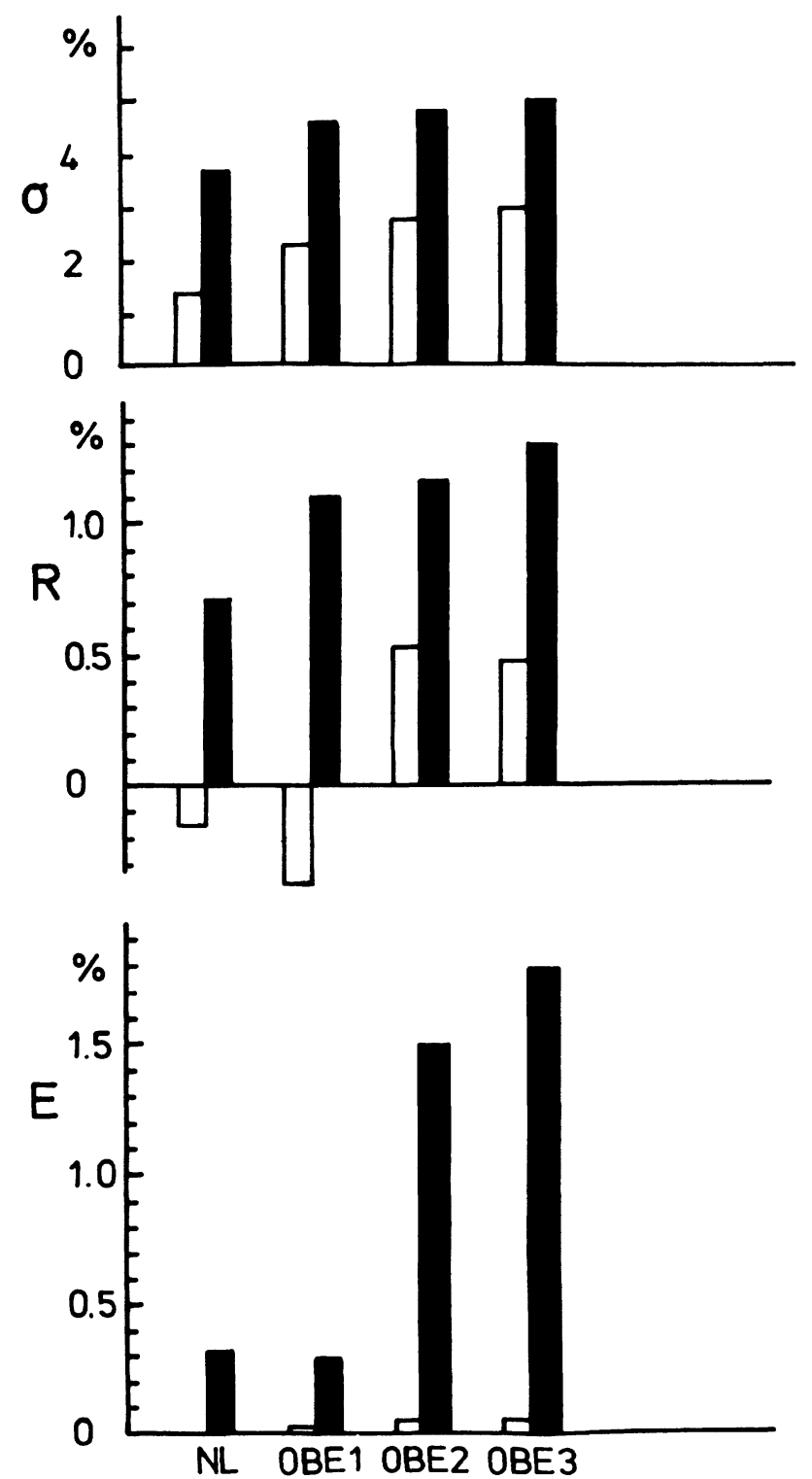

FIG. 8. The average errors (open case) and the rms errors (shaded cases) for the fit observables $E, R$, and $\sigma$ and for the various OBE sets (after fitting the $\sigma$ meson). The result of the "set NL- $Z$ " is given for comparison. 

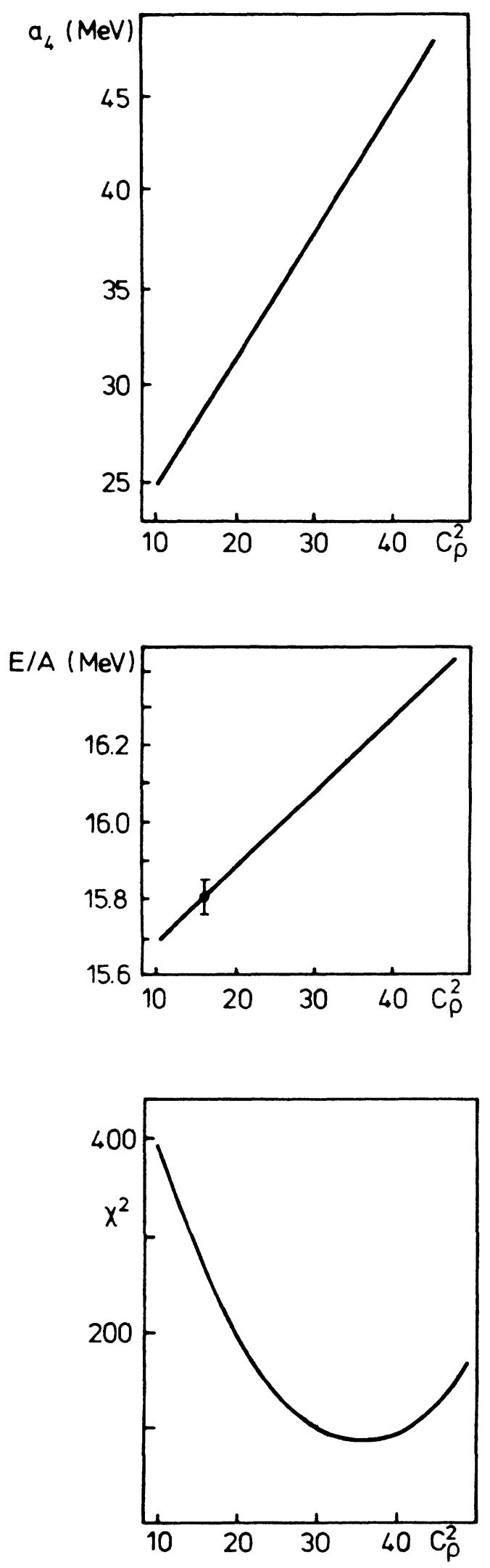

FIG. 9. The quality measure $\chi^{2}$, the binding energy $E / A$ and symmetry energy $a_{4}$ in nuclear matter from fits within the nonlinear model but with fixed $\rho$ coupling, drawn as a function of the effective $\rho$ coupling $c_{\rho}^{2}=\left(g_{\rho} m_{B} / m_{\rho}\right)^{2}$. coupling constant. The results are shown in Fig. 9 as functions of the effective $\rho$ coupling, $c_{\rho}^{2}=\left(g_{\rho} m_{B} / m_{\rho}\right)^{2}$. The $\chi^{2}$ demonstrates the need for a $\rho$ meson, and it clearly displays the tendency to rather large $\rho$ couplings compared with the $c_{\rho}^{2}$ values in the OBEP's. These are necessary to properly adjust the isotopic trends in binding and radius.

From the observables we show in Fig. 9, there are only two which depend significantly on $c_{\rho}^{2}$. Not surprisingly, the most dramatic effect is seen on the asymmetry energy coefficient, $a_{4}$. We see that the large $\rho$ coupling gives rise also to a too large $a_{4}$ and a slightly too large binding energy, $E / A$. This is all a bit puzzling. One needs to study excitation data in order to see whether the large $c_{\rho}^{2}$ is compatible with isovector giant resonances. ${ }^{33}$

\section{F. The effect of the nonlinear coupling $b_{3}$}

In Secs. VI A and VI B, we have seen that the nonlinear self-couplings of the $\sigma$ meson are very important to achieve a decent reproduction of nuclear ground-state properties. It is interesting to see how the results depend on the strength of the nonlinearities. Of course, it is inconvenient to trace dependencies on two parameters. Thus we investigate the effect of the quartic self-coupling $b_{3}$. This is done again by performing fits within the nonlinear model, but keeping $b_{3}$ fixed. The results are drawn in Fig. 10 as functions of the effective coupling $B_{3}=b_{3} / g_{\sigma}^{4}$.

$\chi^{2}$ displays a sharp minimum at the optimal value of $B_{3}$ from set NL- $Z$. Here, $B_{3}$ is negative and there is little chance for a second minimum with positive $B_{3}$. A negative $B_{3}$ makes the equation of state for the $\sigma$ field asymptotically unstable. This, of course, would be disastrous if we believed the Lagrangian to be an $a b$ initio microscopic Lagrangian. For then, any quantum fluctuation of the $\sigma$ field would trigger a tunneling to the unstable region. However, since we are working with an effective Lagrangian for use in connection with the mean-field approximation, this is not a problem. Here the $\sigma$ field is a classical field, and it cannot overcome the high barrier in the equation of state. Thus, we think that the negative $B_{3}$ is not a point of concern if one stays within the limits of the model. One has to be careful, however, in applying the parametrization to a dynamical calculation; if the system acquires sufficient excitation energy, the $\sigma$ field may be kicked over the barrier and run to infinite values. Here is a point where we urgently need more theoretical development to get definite statements about higher nonlinearities, which could render the equation of state stable, or about an energy dependence of $b_{2}$ and $b_{3}$, which stabilizes the $\sigma$ field for energetic processes.

In Fig. 10, we also show the results for those observables which depend sensitively on $B_{3}$. Most interesting is the strong variation of the compressibility $K$. Small $B_{3}$ gives too large a compressibility. There is a clear relation between $K$ and the average surface thickness, small surfaces correspond to large $K$, and vice versa. This connection was also observed in classical mean-field models. ${ }^{21}$ 

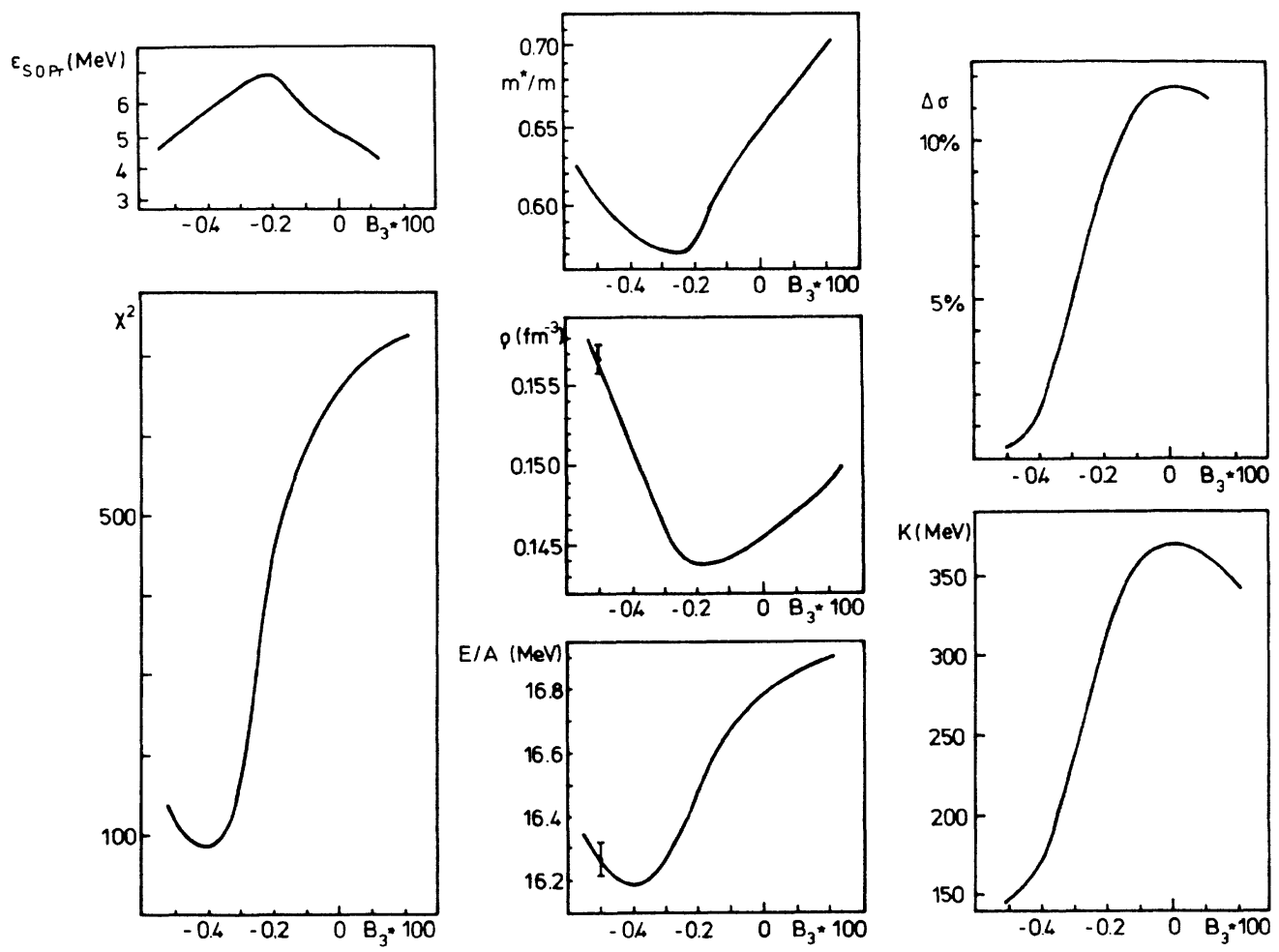

FIG. 10. The quality measure $\chi^{2}$, the nuclear matter properties $E / A, \rho_{0}, K$, and $m^{*} / m$, the spin-orbit splitting in ${ }^{16} \mathrm{O} \delta \epsilon_{\mathrm{s} .0}$, and the average deviation of the surface thickness $\Delta \sigma=\left(\sigma^{\exp }-\sigma^{\text {th }}\right) / \sigma^{\exp }$ for fits within the nonlinear model but with fixed $B_{3}$, drawn versus the effective quartic self-coupling $B_{3}=b_{3} / g_{\sigma}^{4}$.

The trends in $E / A$ and $\rho_{0}$ favor the solution with $B_{3} \approx-0.4$. For larger values of $B_{3}$, these observables deviate even more from the local density model (LDM) values. The spin-orbit splitting has a maximum at $B_{3}=-0.2$ and decreases monotonically with rising $B_{3}$. This is an additional hint that positive values of $B_{3}$ are not desirable. Finally we see that the effective mass $m^{*} / m$ undergoes strong changes. We will discuss this topic in Sec. VI G.

\section{G. Constraining the effective mass}

The fits in the linear and in the nonlinear models all have very small effective masses, $m^{*} / m \approx 0.58$, or even lower. With the nonrelativistic Skyrme force, one usually obtains values around 0.8 . This is a large difference, even in view of the fact that we are comparing different definitions of the effective mass. The small effective masses cause some problems with the stability of relativistic calculations and are probably nonphysical. Also, one obtains an unstable equation of state for neutron star matter. ${ }^{42}$ In Fig. 11, we show the particle and antiparticle potential for ${ }^{40} \mathrm{Ca}$ evaluated with set NL-Z. We see that the shell fluctuations diminish the gap between the particle and antiparticle potential to a value below the average gap $2 m^{*}$. A small enhancement of the central density generates a situation where the antiparticle potential crosses the $1 s_{1 / 2}$ particle state, and then the model

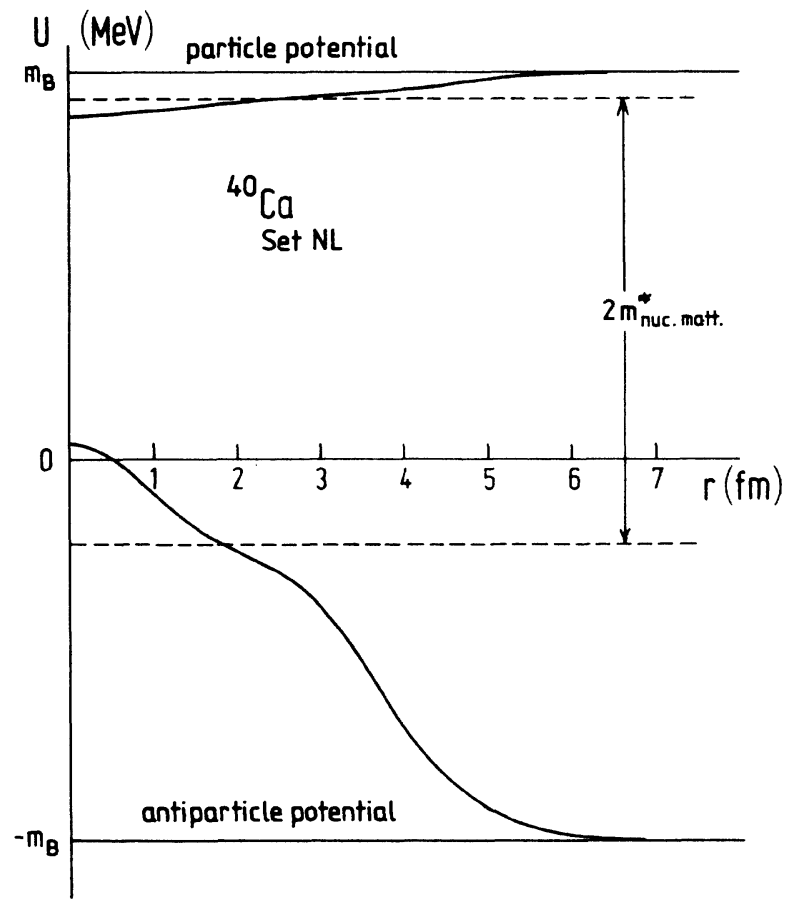

FIG. 11. The particle-potential, $U_{\text {scalar }}+U_{\text {vector }}+m_{B}$, and the antiparticle potential, $-m_{B}-U_{\text {scalar }}+U_{\text {vector }}$, for neutrons in ${ }^{40} \mathrm{Ca}$ evaluated for "set NL-Z" and drawn as functions of the radial distance $r$. 
becomes unstable with respect to excitations out of the vacuum.

We have performed fits within the nonlinear model to the standard set of data, plus a contribution to $\chi^{2}$ which enforces a desired value of $\mathrm{m}^{*} / \mathrm{m}$ in nuclear matter. The result is shown as the straight line in Fig. 12. We see that the $\chi^{2}$ clearly favors $m^{*} / m \approx 0.58$. Values around 0.60 may be acceptable if a somewhat increased stability is desired. However, larger values for $m^{*} / m$ are clearly excluded. It is not only the $\chi^{2}$ which rises dramatically with rising $m^{*} / m$, but also the spin-orbit splitting $\delta \epsilon_{\mathrm{s} \text {. }}$. is decreasing substantially. The proper value of $\delta \epsilon_{\mathrm{s} . o^{*}}$. seems to depend critically on large scalar and vector fields which cancel to yield a small nucleon potential, but which also yield very low effective masses. We also have performed fits which include, as additional data, the $\delta \epsilon_{\mathrm{s} \text {. o. }}^{P}$ and $\delta \epsilon_{\mathrm{s.o}}^{N}$. in ${ }^{16} \mathrm{O}$, the dashed line in Fig. 12. Obviously, there is no chance to decouple $m^{*} / m$ and $\delta \epsilon_{\text {s.o. }}$ within the given model.

In Fig. 12, we show also those nuclear matter properties which display sufficient variation with $m^{*} / m$. It is comforting to see that the values which come closest to the commonly believed nuclear matter properties (see line LDM in Table V) occur just at low $m^{*} / m$. We see, furthermore, a surprising sensitivity of the extrapolation to nuclear matter with the inclusion of the extra data, $\delta \epsilon_{\text {s.o. }}$, in the fit. This shows the degree of ambiguity in all those extrapolations to nuclear matter.
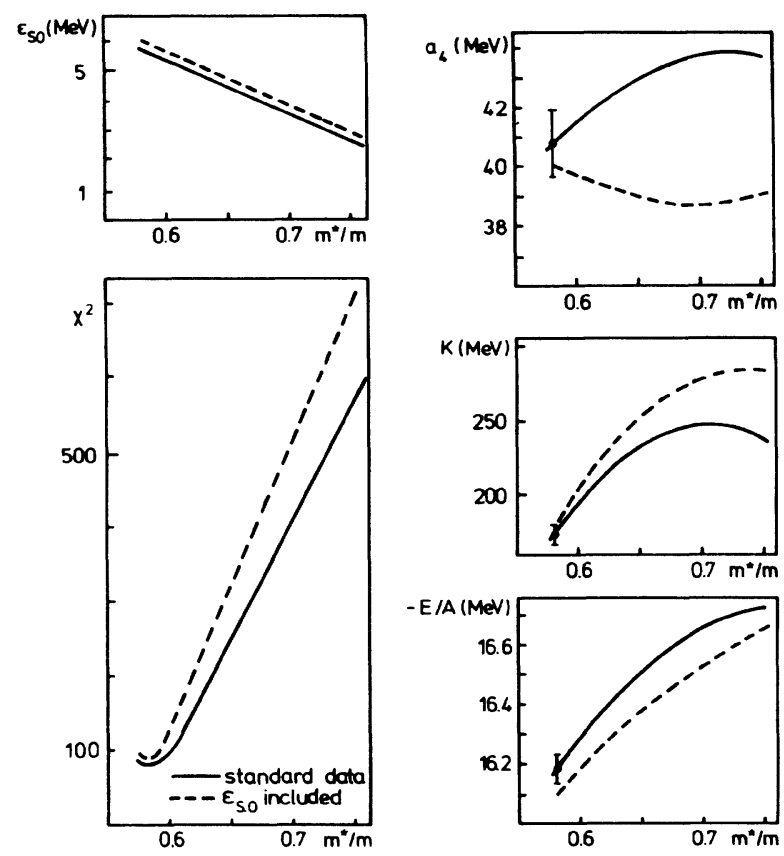

FIG. 12. The quality measured $\chi^{2}$, the nuclear matter properties $E / A, K$, and $a_{4}$, and the spin-orbit splitting in ${ }^{16} \mathrm{O} \delta \epsilon_{\mathrm{s.o}}$. for fits within the nonlinear model to the standard set of data and with constraint on a certain effective mass $m^{*} / m$, drawn as a function of this $m^{*} / m$. In addition to the fit with standard data (straight line), we also give the results of fits to the standard data plus the spin-orbit splitting $\delta \epsilon_{\text {s.o. }}$ as an extra datum in $\chi^{2}$, weighted with an average error of $0.3 \mathrm{MeV}$ (dashed line).

\section{H. Varying the nucleon radius}

As pointed out in Sec. IV, there are already some correlation effects taken into account in the evaluation of the charge density: the folding with the charge and magnetic density distributions of the proton and the neutron. This is certainly an important effect, and it cannot be neglected. However, the uncertainty in this procedure is that free-nucleon form factors are used for bound nucleons which move off the mass shell. In the same context, there are investigations which estimate the quark structure of nucleons moving in a nuclear medium and which conclude that the nucleon radius should increase. ${ }^{43}$ Thus, we have tried to check the effect of a changing nucleon radius.

To this end, we have simply scaled the intrinsic nucleon form factor by a factor $(1+\eta)$

$$
F_{i}(q) \rightarrow F_{i}[(1+\eta) q]
$$

which means that the nucleon radii are also increased by a factor $(1+\eta)$. For a fixed increase $\eta$, we have performed fits to the standard data within the nonlinear model. The results as functions of the percent increase $\eta$ are shown in Fig. 13. The effect of the increased radius on $\chi^{2}$ is dramatic. However, we see that the fits with the corrected surface thickness $\sigma_{0}$, dashed line in Fig. 13, are less biased towards larger nucleon radii, and it is not yet clear what would happen if all collective surface modes were included in the correction of $\sigma$. Here we have two effects which soften the surface: collective surface modes and an increase of the nucleon radius.

In Fig. 13, we have also shown those nuclear matter properties which depend sensitively on the nucleon radius. The trend towards smaller $\rho_{0}$ is understandable: the nucleon radius becomes larger and less extension
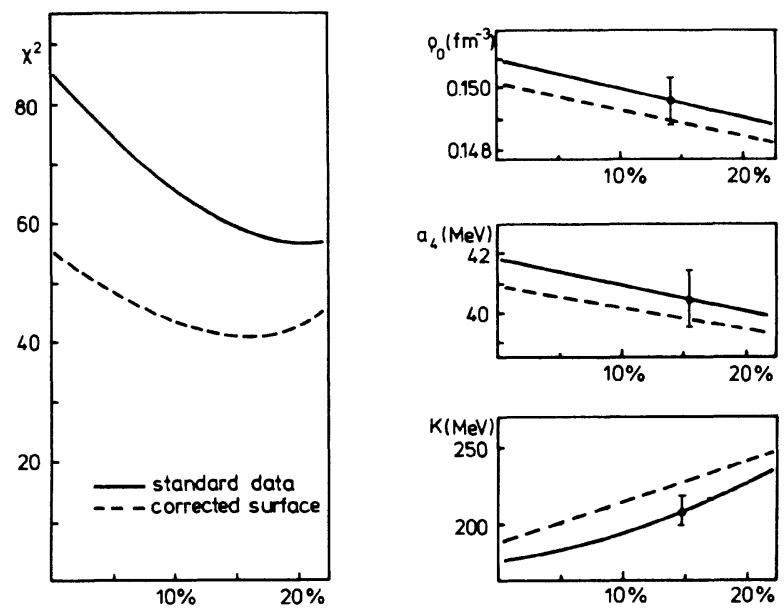

FIG. 13. The quality measure $\chi^{2}$ and the nuclear matter properties $K, a_{4}$ and $\rho_{0}$ from fits within the nonlinear model with the intrinsic nucleon radius increased by $\eta$. Results are drawn as a function of the radius increase $\eta$ in percent. Fits are performed once to the standard set of data (straight line) and once to the set of data with corrected surface thickness $\sigma_{0}$ (dashed line). 
needs to be provided by the mean-field solution. The effect on $K$ is also clear from the correlation between compressibility $K$ and surface thickness $\sigma$ already observed: smaller $\sigma$ correspond to larger $K$, and an increase of the nucleon allows smaller $\sigma$ in the mean-field solution. It is interesting to note that there is also an effect on the surface symmetry $a_{4}$. All three trends are very desirable and bring these nuclear matter properties closer to the LDM values (see Table V).

\section{The effect of tensor couplings}

As a first extension beyond a minimal coupling theory, we have introduced tensor couplings for the $\rho$ meson and the $\omega$ meson in the effective Lagrangian (1). This is a useful extension for a comparison with $O B E$ potentials because these already include such tensor couplings (see Sec. VID). This coupling adds some momentum and spin dependence, and it is interesting to see how this modifies the model.

First we have examined the sensitivity to a tensor coupling for the $\rho$ meson. In Fig. 14, we have given $\chi^{2}$ its individual contributions, and the surface symmetry coefficient $a_{4}$ as a function of the relative $\rho$ coupling $f_{\rho} / g_{\rho}$. We see that nothing is gained by the $\rho$ tensor coupling. It is just insensitive for a broad range of $f_{\rho} / g_{\rho}$. The nuclear matter properties are also rather insensitive, as illustrated by $a_{4}$ in Fig. 14. It is interesting to see, however, that the single contributions to $\chi^{2}$ show more dependence on $f_{\rho} / g_{\rho}$. The surfaces seem to like tensor coupling, whereas energies and radii do not.

Next we investigated the tensor coupling for the $\omega$ meson. In Fig. 15, we have drawn the $\chi^{2}$ and the nuclear matter properties $K$ and $m^{*} / m$ as functions of $f_{\omega} / g_{\omega}$. Here we see some improvement in $\chi^{2}$. Most of the observables are insensitive, and they do not change significantly within their extrapolation errors. However, the compressibility decreases further with decreasing $f_{\omega}$, and $m^{*} / m$ increases. The larger $m^{*} / m$ is a desirable feature and is achieved without destroying the good spin-orbit splitting. This is an important result and should be included in future studies with relativistic mean-field models.

\section{CONCLUSIONS}

We have investigated the strengths and the weaknesses of a relativistic mean-field description of the ground state of nuclei. The theory is considered to be the relativistic generalization of a nonrelativistic mean-field theory employing effective forces like the Skyrme force. In the relativistic case, forces are generalized to include meson degrees of freedom. Thus, we work with a seemingly basic Lagrangian of coupled mesons and nucleons. This Lagrangian, however, is an effective Lagrangian, and the meson parameters are the free parameters of the model.

We have studied systematically the possibilities and the limits of the model by using least-squares fits to nuclear ground-state properties; namely to binding energy $E$, diffraction radius $R$, and surface thickness $\sigma$ for eight nuclei. The energy is the least problematic quantity because it can be derived consistently from the given effective Lagrangian. Other observables are more ambiguous since one should derive for them effective operators which include the same type of correlations which have been assumed in the effective Lagrangian. These correlations are not known in detail. We have chosen, with diffraction ra-
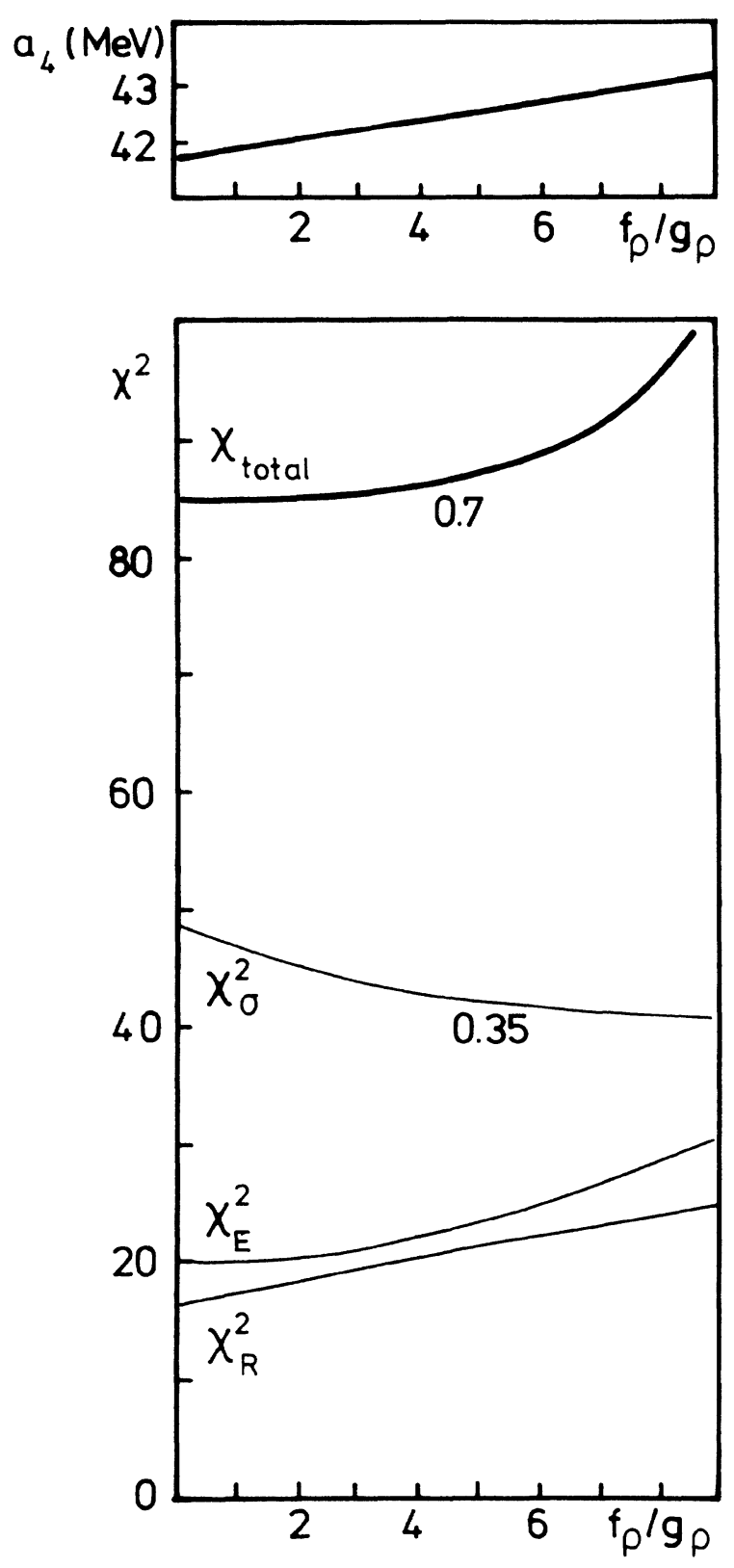

FIG. 14. The quality measure $\chi^{2}$ and the symmetry energy $a_{4}$ from fits to standard data within the tensor-extracted model. Fits are done for fixed-tensor couplings $f_{\rho}$ and $f_{\omega}=0$, minimizing $\chi^{2}$ with respect to all other free parameters. Results are drawn as a function of the $\rho$-tensor coupling $f_{\rho} / g_{\rho}$. For $\chi^{2}$ we also show the three partial contributions coming from energy $E$, radius $R$, and surface thickness $\sigma$. 
dius $R$ and surface thickness $\sigma$, those parameters of the charge form factor which are least sensitive to correlations. However, certain corrections which go beyond the mean field are already included: the center-of-mass correction to the binding energy, the form factor, and also the folding of the charge density with the intrinsic electric and magnetic nucleon form factor. These are unavoidable and rather clear corrections. Additional insight into properties of the model is gained by looking at a few other observables which are either not experimentally accessible or which are shadowed by correlation effects.

We find that the linear model is insufficient for a reasonable description of nuclei. The nonlinear model,
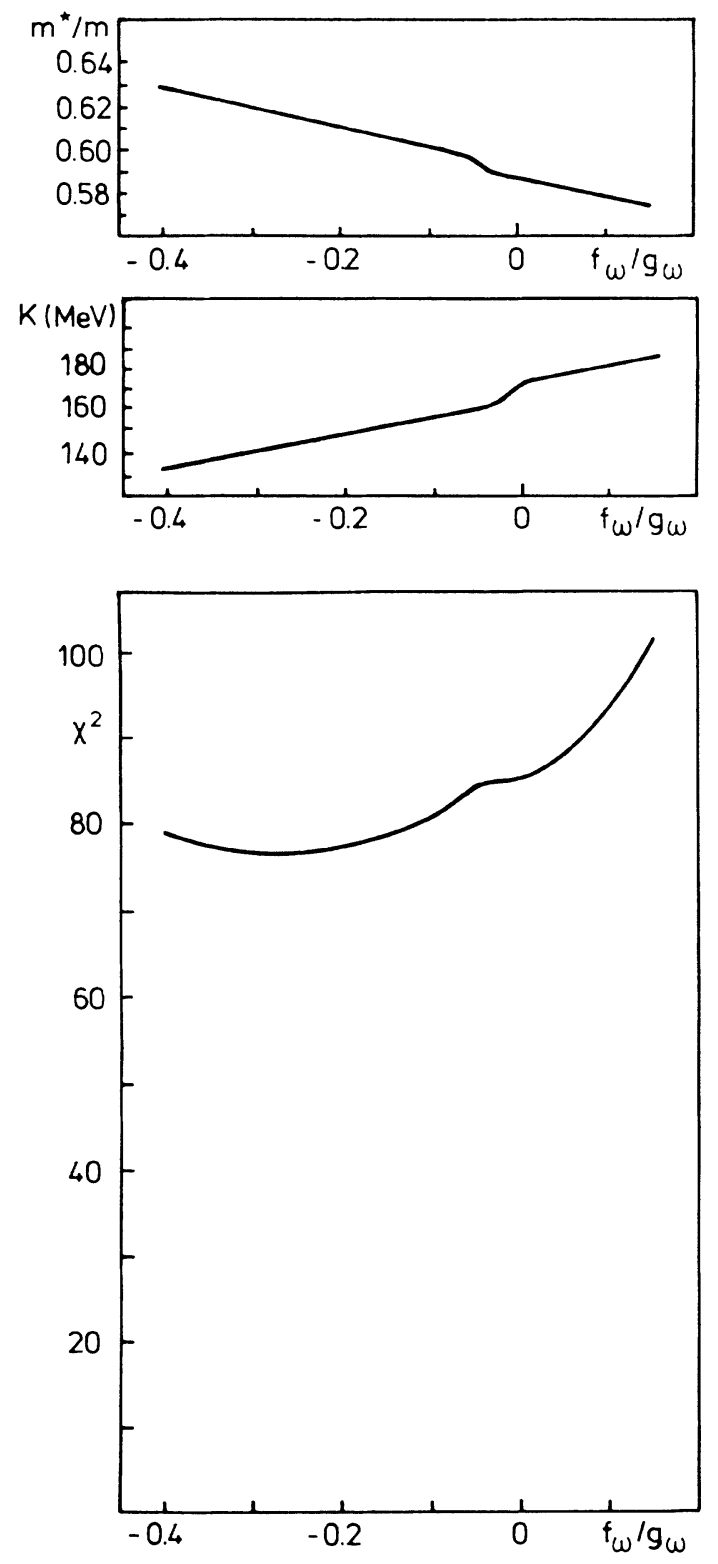

FIG. 15. The quality measure $\chi^{2}$ and the nuclear matter properties effective mass $m^{*} / m$ and compressibility $K$ from fits to standard data within the extended model. The tensor couplings have been kept fixed during the fit, $f_{\rho}$ at $f_{\rho}=0$ and $f_{\omega}$ at various values. Results are plotted versus $f_{\omega} / g_{\omega}$. on the other hand, gives a very good description with an error of $0.3 \%$ for the energies, of $0.7 \%$ for the radii, and of $3.7 \%$ for the surfaces. This description reaches the quality of the Skyrme forces with the additional bonus that the spin-orbit force and other spin properties are given without extra adjustment. This nonlinear model with this parametrization provides a good starting point for any further investigation with the relativistic meanfield theory.

The inclusion of tensor couplings improves a bit the reproduction of surfaces. We have to keep in mind, however, that the choice of tensor couplings as the next extension is somewhat arbitrary, guided again by a similarity to OBE potentials. The nonlinear self-couplings of the scalar meson are an essential ingredient which brings the model to quantitative agreement with data. One problem is that the parameters of the nonlinear terms are such that the meson equation of state is asymptotically unstable. We have studied systematic variations of the nonlinear coupling and found no chance to construct an asymptotically stable equation of state. We think that this possible instability is no problem within a mean-field treatment since the unstable region is separated by a barrier which cannot be penetrated by a classical meson field. However, problems may occur if one applies the parametrization to dynamical calculations.

All the parametrizations which we find lead to low effective masses, $m^{*} / m \approx 0.58$. This causes problems in extrapolations of the model to neutron matter, high densities, and high temperatures, and it yields instabilities for some nuclei with large density fluctuations.

We have also studied, in a preliminary fashion, two correlation effects: the influence of quadrupole surface vibrations and an increase of the nucleon radius in the nuclear medium. Although very different in origin, both effects act in the same direction increasing the surface thickness. This increase is very desirable. It improves substantially the quality of the reproduction of the nuclear surface. The optimal parametrization found by our fits provides a good basis for further studies in this direction. We conclude that the nonlinear relativistic meanfield model, with the appropriate choice of parameters, allows a very good description of nuclear ground-state properties, competitive with and, in some sense, superior to the Skyrme force models. We clearly reach the limits of the parametrization and perhaps of the mean-field approximation, as such. Further investigations should look for more correlation effects (surface modes). They should include more observables, e.g., dynamic properties, in order to critically explore the parametrization. Also, one needs more theoretical development which draws the lines from a microscopic Lagrangian to the effective mean-field Lagrangian. For many of these investigations, the optimal parametrization presented in this paper provides a good starting point.

\section{ACKNOWLEDGMENTS}

The Joint Institute for Heavy Ion Research has as member institutions the University of Tennessee, Vanderbilt University, and the Oak Ridge National Laboratory. 
It is supported by the members and by the Department of Energy through Contract No. DE-AS05-76ER0-4936 with the University of Tennessee. Oak Ridge National Laboratory is operated by the Division of Nuclear Physics, U.S. Department of Energy under Contract No. DEAC05-840R21400 with Martin Marietta Energy Systems, Inc.

\section{APPENDIX: SPHERICAL REPRESENTATION AND NUMERICAL PROCEDURE}

We restrict the present considerations to the case of spherically symmetric mean fields, i.e., $\Phi=\Phi(|r|)$, $V_{0}=V_{0}(|r|)$, etc. The nucleon wave function can then be expressed as ${ }^{23}$

$$
\phi_{\alpha}=\left(\begin{array}{l}
i \frac{G_{\alpha}(r)}{r} \mathcal{Y}_{j_{\alpha} l_{\alpha} m_{\alpha}} \\
\frac{F_{\alpha}(r)}{r} \frac{\sigma \cdot p}{r} Y_{j_{\alpha} l_{\alpha} m_{\alpha}}
\end{array}\right),
$$

where $\mathcal{Y}_{j l m}$ is the spinor spherical harmonics and $G_{\alpha}$ and
$F_{\alpha}$ are the remaining radial wave functions for upper and lower components. They are normalized so that

$$
\int_{0}^{\infty} d r\left\{\left|G_{\alpha}\right|^{2}+\left|F_{\alpha}\right|^{2}\right\}=1 \text {. }
$$

With the above choice of relative phases, both $G_{\alpha}$ and $F_{\alpha}$ are real. The densities then become

$$
\begin{aligned}
& \rho_{s}=\frac{1}{4 \pi r^{2}} \sum_{\alpha} w_{\alpha}\left(2 j_{\alpha}+1\right)\left(G_{\alpha}^{2}-F_{\alpha}^{2}\right), \\
& \rho_{0}=\frac{1}{4 \pi r^{2}} \sum_{\alpha} w_{\alpha}\left(2 j_{\alpha}+1\right)\left(G_{\alpha}^{2}+F_{\alpha}^{2}\right), \\
& \rho_{0,0}=\frac{1}{4 \pi r^{2}} \sum_{\alpha} w_{\alpha}\left(2 j_{\alpha}+1\right) \tau_{0 \alpha}\left(G_{\alpha}^{2}+F_{\alpha}^{1}\right), \\
& \rho_{P, 0}=\frac{1}{2}\left(\rho_{0}+\rho_{0,0}\right), \\
& \rho_{0}^{T}=\frac{1}{4 \pi r^{2}} \sum_{\alpha} w_{\alpha}\left(2 j_{\alpha}+1\right) 2 \partial_{r}\left(F_{\alpha} G_{\alpha}\right), \\
& \rho_{0,0}^{T}=\frac{i}{4 \pi r^{2}} \sum_{\alpha} w_{\alpha}\left(2 j_{\alpha}+1\right) 2 \partial_{r}\left(F_{\alpha} G_{\alpha}\right) \tau_{0 \alpha} .
\end{aligned}
$$

The meson field equations become simply radial Laplace equations. The nuclear radial wave functions are determined by the coupled equations

$$
\begin{aligned}
& \epsilon_{\alpha} G_{\alpha}=\left(-\frac{d}{d r}+\frac{\kappa_{\alpha}}{r}+\frac{f_{V}}{2 m} \partial_{r} V_{0}+\frac{f_{R}}{4 m_{B}} \partial_{r} R_{0} \tau_{0, \alpha}\right) F_{\alpha}+\left(m_{B}+g_{S} \Phi+g_{V} V_{0}+\frac{1}{2} g_{R} R_{0,0} \tau_{0, \alpha}+e A_{0} \frac{1+\tau_{0 \alpha}}{2}\right) G_{\alpha}, \\
& \epsilon_{\alpha} F_{\alpha}=\left(\frac{d}{d r}+\frac{\kappa_{\alpha}}{r}+\frac{f_{V}}{2 m} \partial_{r} V_{0}+\frac{f_{R}}{4 m_{B}} \partial_{r} R_{0} \tau_{0, \alpha}\right) G_{\alpha}-\left(m_{B}+g_{S} \Phi-g_{V} V_{0}-\frac{1}{2} g_{R} R_{0,0} \tau_{0 \alpha}-e A_{0} \frac{1+\tau_{0 \alpha}}{2}\right) F_{\alpha},
\end{aligned}
$$

where

$$
\kappa_{\alpha}= \begin{cases}-(j+1 / 2) & \text { for } j=1+1 / 2 \\ +(j+1 / 2) & \text { for } j=1-1 / 2\end{cases}
$$

These equations are solved numerically by standard techniques from conventional Skyrme-Hartree-Fock. To this end, we transform Eq. (A4a) to resemble a Schrödinger equation. This is achieved by eliminating $F_{\alpha}$. We obtain as equation for $G_{\alpha}$

$\epsilon_{\alpha} G_{\alpha}=-\left(\frac{d}{d r}-W(r)\right) M_{\mathrm{eff}}^{-1}\left[\frac{d}{d r}+W(r)\right) G_{\alpha}+U_{\mathrm{eff}} G_{\alpha}$,

(A5a)

$M_{\mathrm{eff}}=\epsilon_{\alpha}+m_{B}+g_{S} \Phi$

$$
-g_{V} V_{0}-\frac{1}{2} g_{R} R_{0,0} \tau_{0 \alpha}-e A_{0} \frac{1+\tau_{0 \alpha}}{2},
$$

$U_{\mathrm{eff}}=m_{B} g_{S} \Phi+g_{V} V_{0}+\frac{1}{2} g_{R} R_{0,0} \tau_{0 \alpha}+e A_{0} \frac{1+\tau_{0 \alpha}}{2}$,

(A5c)

$$
W(r)=\frac{\kappa_{\alpha}}{r}+\frac{f_{V}}{2 m} \partial_{r} V+\frac{f_{R}}{4 m} \partial_{r} R_{0} \tau_{0 \alpha} .
$$

The $F_{\alpha}$ is needed for the normalization (A2) and the densities (A3). It is reconstructed from

$$
F_{\alpha}=M_{\mathrm{eff}}^{-1}\left(\frac{d}{d r}+W(r)\right) G_{\alpha}
$$

The effective mass in the "Schrödinger equation" (A5a) depends itself on the single-particle energy $\epsilon_{\alpha}$. This causes no problem since we are, anyway, going to solve Eq. (A5a) iteratively. We have seen that this additional energy dependence does not destroy the stability of the procedure. The effective Schrödinger equation (A5), together with the field equations for the mesons, is solved with an accelerated gradient iteration which is taken over from an existing Skyrme-Hartree-Fock code. The procedure goes as follows. In the $n$th iteration step, we have arrived at a set of wave functions $G_{\alpha}^{(n)}(r)$ and $F_{\alpha}^{(n)}(r)$, occupation numbers $w_{\alpha}^{(n)}$, and a scalar field $\Phi^{(n)}(r)$. First, we add up the densities $\rho_{S}^{(n)}(r), \rho_{0}^{(n)}(r), \rho_{0,0}^{(n)}(r)$, and $\rho_{P r, 0}^{(n)}(r)$ according to Eqs. (A3) and using $G_{\alpha}^{(n)}, F_{\alpha}^{(n)}$, and $w_{\alpha}^{(n)}$. Second, we determine the meson fields by solving the radial Laplace equations 


$$
\begin{aligned}
{\left[-\frac{d^{2}}{d r^{2}}+m_{S}^{2}\right](} & \left.\Phi^{(N+1)}\right) \\
& =-r\left[g_{S} \rho_{S}^{(n)}+b_{2}\left(\Phi^{(n)}\right)^{2}+b_{3}\left(\Phi^{(n)}\right)^{3}\right],
\end{aligned}
$$$$
\left[-\frac{d^{2}}{d r^{2}}+m_{V}^{2}\right)\left(r V_{0}^{(n+1)}\right)=r g_{V} \rho_{0}^{(n)}
$$

and similarly for $R_{00}^{(n+1)}$ and $A_{0}^{(n+1)}$. Third, we combine the meson fields to $M_{\mathrm{eff}}^{(n+1)}$ and $U_{\mathrm{eff}}^{(n+1)}$, as given in (A5), and iterate the $G_{\alpha}$ by an accelerated gradient step

$$
\begin{aligned}
G_{\alpha}^{(n+1)}=\mathcal{O} & {\left[\left\lfloor-\frac{d}{d r}+W(r)\right]\left(M_{\mathrm{eff}}^{(n+1)}\right)^{-1}\left[\frac{d}{d r}+W(r)\right]\right.} \\
& \left.+\left(U_{\mathrm{eff}}^{(n+1)}-\epsilon_{\alpha}^{(n)}\right) G_{\alpha}^{(n)}\right],
\end{aligned}
$$

where $\mathcal{O}$ means orthonormalization of all $G_{\alpha}$ such that

$d r\left(G_{\alpha}^{(n+1)}+G_{\beta}^{(n+1)}+F_{\alpha}^{(n+1)}+F_{\beta}^{(n+1)}\right)=\delta_{\alpha \beta}$.

Accordingly, $F_{\alpha}^{(n+1)}$ are evaluated from the $G_{\alpha}^{(n+1)}$ using (A5d) with $M_{\mathrm{eff}}^{(n+1)}$. Fourth, we evaluate new $\epsilon_{\alpha}$ by averaging the Dirac equation

$$
\epsilon_{\alpha}^{(n+1)}=\int_{0}^{\infty} d r\left[F_{\alpha}^{\dagger}\left[\frac{d}{d r}+W(r)\right] G_{\alpha}+G_{\alpha}^{\dagger}\left[-\frac{d}{d r}+W(r)\right] F_{\alpha}+G_{\alpha}^{\dagger} U_{\mathrm{eff}} G_{\alpha}-F_{\alpha}^{\dagger}\left(M_{\mathrm{eff}}-\epsilon_{\alpha}^{(n)}\right) F_{\alpha}\right]^{(n+1)}
$$

where every quantity on the right-hand side bears iteration index $(n+1)$. Fifth, we determine the $w_{\alpha}^{(n+1)}$ according to the $\epsilon_{\alpha}^{(n+1)}$ by adjusting a Fermi surface $\epsilon_{\text {Fermi }}^{(n+1)}$ such that the particle number is given with (A5c). This completes one iteration step. The iteration is continued until the binding energy is stable up to six digits. The wave functions and the fields are represented on a grid in $r$ space with a spacing of $r=0.3 \mathrm{fm}$ and an extension up to $R_{\max }=1.16 \mathrm{fm} \mathrm{A}^{1 / 3}+6.4 \mathrm{fm}$. We use five-point formulas for integration and differentiation. The Laplace equations are solved with a scheme which achieves fivepoint precision for the Laplacian by using only threepoint connections. Altogether we obtain a very fast code which allows us to run it over and over again a leastsquares fit.
*Permanent address: Institut für Theoretische Physik, Universität Erlangen, D-8520 Erlangen, Federal Republic of Germany.

†Permanent address: Institut für Theoretische Physik, Universität Frankfurt, D-6000 Frankfurt am Main, Federal Republic of Germany.

${ }^{1}$ P. Quentin and H. Flocard, Ann. Rev. Nucl. Part. Sci. 28, 523 (1978).

${ }^{2}$ J. W. Negele, Rev. Mod. Phys. 54, 913 (1982).

${ }^{3}$ Time Dependent Hartree-Fock and Beyond, Vol. 171 of Lecture Notes in Physics, edited by K. Goeke and P.-G. Reinhard (Springer-Verlag, Berlin, 1982).

${ }^{4}$ K. T. R. Davies, K. R. S. Devi, S. E. Koonin, and M. R. Strayer, in Treatise on Heavy-Ion Science, edited by D. A. Bromley (Plenum, New York, 1985), Vol. 3.

${ }^{5}$ L. D. Miller and A. E. S. Green, Phys. Rev. C 5, 241 (1972).

6J. D. Walecka, Ann. Phys. 83, 491 (1974).

${ }^{7}$ J. Boguta and A. R. Bodmer, Nucl. Phys. A292, 414 (1977).

${ }^{8}$ R. Brockmann, Phys. Rev. C 18, 1510 (1978).

${ }^{9}$ C. J. Horowitz and B. D. Serot, Nucl. Phys. A368, 503 (1981).

${ }^{10}$ J. Boguta, Phys. Lett. 106B, 247 (1987); 106B, 245 (1987); 106B, 250 (1987).

${ }^{11}$ J. Boguta and H. Stöcker, Phys. Lett. 120B, 289 (1983).

${ }^{12}$ A. Bouyssy, S. Marcos and Pham von Thieu, Nucl. Phys. A422, 541 (1984).

${ }^{13}$ P.-G. Reinhard, M. Rufa, J. Maruhn, W. Greiner, and J. Friedrich, Z. Phys. A 323, 13 (1986).

${ }^{14}$ B. D. Serot and J. D. Walecka, Adv. Nucl. Phys. 15 (1987).

${ }^{15}$ L. G. Arnold, B. C. Clark, R. L. Mercer, and P. Schwandt, Phys. Rev. C 23, 1949 (1981).
${ }^{16}$ R. Brockmann and R. Machleidt, Phys. Lett. 149B, 283 (1984).

${ }^{17}$ B. TerHaar and R. Malfliet, Phys. Lett. B 172, 10 (1986).

18J. W. Negele and D. Vautherin, Phys. Rev. C 5, 1472 (1972).

${ }^{19}$ S. A. Chin, Ann. Phys. 108, 301 (1977).

${ }^{20}$ K. Holinde, Phys. Rev. 68, 122 (1981).

${ }^{21}$ J. Friedrich and P.-G. Reinhard, Phys. Rev. C 33, 335 (1986).

${ }^{22}$ P.-G. Reinhard, M. Rufa, and J. Maruhn, W. Greiner, and J. Friedrich, Z. Phys. A 323, 13 (1986).

${ }^{23}$ J. D. Bjorken and S. D. Drell, Relativistic Quantum Mechanics (McGraw-Hill, New York, 1964).

${ }^{24}$ J. D. Bjorken and S. D. Drell, Relativistic Quantum Field Theory (McGraw-Hill, New York, 1965).

25J. Blocki and M. Flocard, Nucl. Phys. A273, 45 (1976).

${ }^{26}$ A. L. Fetter and J. D. Walecka, Quantum Theory of ManyParticle Systems (McGraw-Hill, New York, 1971), p. 171.

${ }^{27}$ B. D. Day, Rev. Mod. Phys. 50, 495 (1978).

${ }^{28}$ B. Dreher, J. Friedrich, K. Merle, G. Lührs, and H. Rothaas, Nucl. Phys. A235, 219 (1974).

${ }^{29}$ G. G. Simon, C. Schmitt, F. Borkowski, and V. H. Walther, Nucl. Phys. A333, 318 (1980); V. H. Walther, private communication.

30J. L. Friar and J. W. Negele, Adv. Nucl. Phys. 8, 219 (1975).

31J. Friedrich and N. Voegler, Nucl. Phys. A373, 191 (1982).

${ }^{32}$ P.-G. Reinhard and D. Drechsel, Z. Phys. A 290, 85 (1979).

${ }^{33}$ P.-G. Reinhard and J. Friedrich, Z. Phys. A 321, 619 (1985).

${ }^{34}$ R. A. Broglia, O. Hansen, and C. Riedel, Adv. Nucl. Phys. 6, 287 (1973).

${ }^{35}$ P. M. Endt, At. Nucl. Data Tables 23, 3 (1979); 26, 47 (1981).

${ }^{36}$ P. R. Bevington, Data Reduction and Error Analysis for the 
Physical Sciences (McGraw-Hill, New York, 1969).

${ }^{37}$ D. Vautherin and D. M. Brink, Phys. Rev. C 5, 626 (1972).

${ }^{38}$ P.-G. Reinhard, J. Friedrich and N. Voegler, Z. Phys. A 316 347 (1984).

${ }^{39}$ G. E. Brown, in Giant Multipole Resonances, edited by F. E. Bertrand (Harwood-Academic, New York, 1979).
${ }^{40}$ W. D. Myers, At. Nucl. Data Tables 17, 411 (1976).

${ }^{41}$ K. Erkelenz, Phys. Rep. C 13, 191 (1974).

${ }^{42} \mathrm{~N}$. K. Glendenning, private communication.

${ }^{43}$ M. R. Anastasio, L. S. Calenza, W. S. Pong, and C. M. Shakin, Phys. Rep. C 100, 327 (1983). 